\title{
Small Extracellular Vesicles From Human Adipose- derived Stem Cells: A Potential Promoter of Keeping Fat Graft Survival
}

\section{Aizhen Chen}

Fujian Medical University Union Hospital

\section{Shijie Tang}

Fujian Medical University Union Hospital

Jiawei He

Fujian Medical University Union Hospital

\section{Xiangyu Li}

Fujian Medical University Union Hospital

\section{Guohao Peng}

Fujian Medical University Union Hospital

\section{Haoruo Zhang}

Fujian Medical University Union Hospital

\section{Jinghua Chen}

Fujian Medical University

\section{Liangwan Chen}

Fujian Medical University Union Hospital

Xiaosong Chen ( $\square$ chenxiaosong74@163.com )

Fujian Medical University Union Hospital https://orcid.org/0000-0002-3780-5510

\section{Research}

Keywords: Small extracellular vesicles, Human adipose-derived stem cells, Fat graft, angiogenesis

Posted Date: December 16th, 2020

DOI: https://doi.org/10.21203/rs.3.rs-127314/v1

License: (c) (i) This work is licensed under a Creative Commons Attribution 4.0 International License. Read Full License 


\section{Abstract}

Background: Small extracellular vesicles (sEVs) with genetic information secreted by cells play a crucial role in the cellular microenvironment. In this study, our purpose is to explore the characteristics of the small extracellular vesicles of human adipose-derived stem cells (hADSC-sEVs) and studied the role of hADSC-sEVs in improving the survival rate of grafted fat.

Methods: In the present study, we used the transmission electron microscopy, nano-tracking analysis, nanoflow surface protein analysis, zeta potential value to identify sEVs. SEVs' trajectory was traced dynamically to verify whether hADSC-sEVs can be internalized into human umbilical vein endothelial cells (HUVECs) in vitro. The angiogenic property of hADSC-sEVs was observed by measuring the volume, weight and histological analysis of the grafted fats in nude mice modles.

Results: Our research showed that the extracellular vesicles were sEVs with double-layer membrane structure and the diameter of which is within $30-150 \mathrm{~nm}$. hADSC-sEVs exert biological influence mainly through internalization into cells. Compared with the control group, the hADSC-sEVs group had a significantly higher survival rate of grafted fat, morphological integrity and a lower degree of inflammation and fibrosis. And immunohistochemistry showed that hADSC-sEVs significantly increased the neovascularisation and the expression of CD34, VEGFR2 and $\mathrm{KI}-67$ in the graft tissue.

Conclusions: As a potential nanomaterial, hADSC-sEVs has been explored in the field of cell-free application of stem cell technology. hADSC-sEVs promoted the survival of grafted fats by promoting the formation of new blood vessels, which is another promising progress in the field of regenerative medicine. We believe that hADSC-sEVs will have a broad application prospect in the field of regenerative medicine in the future.

\section{Background}

Small extracellular vesicles (sEVs) are 30-150 nm membranous vesicles actively released by cells, containing various kinds of the molecule - proteins, mRNA, miRNA, lipids - that are actively being studied as potential biomarkers and play essential roles in intercellular communication [1,2]. They derive from specific cell types, in particular mesenchymal stem/stromal cells (MSCs), offer powerful therapeutic potential, and can provide a novel therapeutic strategy [3]. Remarkably, studies enrolled have revealed that EVs can promote angiogenesis $[4,5]$ and account for the beneficial paracrine effects of hADSCs therapy [6]. In addition, small extracellular vesicles of human adipose-derived stem cells (hADSC-sEVs) can effectively attenuate myocardial ischemia/reperfusion injury [7] and accelerate cutaneous wound healing [8].

In clinical, autologous fat grafting is playing an increasingly important role in the aesthetic and reconstructive field for its extensive utility for soft tissue rejuvenation, volume augmentation, and body contouring. In contrast, the unpredictable fat resorption and low survival rates limit its further application and development $[9,10]$. Mounting data have demonstrated that adequate neovascularization is vital for 
the survival and maintenance of grafted fat [11]. Therefore, many approaches have been developed to promote angiogenesis and improve fat graft retention. Among these, co-transplantation of autologous adipose tissue with adipose-derived stem cells (ADSCs) or stromal vascular fraction (SVF), known as cellassisted lipotransfer (CAL) technique [12], can enhance the survival rates by stimulating angiogenesis through a paracrine effect [13]. Adipose tissue seems to be the most advantageous tissue from which to isolate stem cells because of its abundancy, subcutaneous location, and the need for less invasive techniques [14]. Furthermore, the primary dilemma of stem cell therapy lies in it's difficult to extend clinical applications for its safety concerns $[15,16]$. Given that, we turned to explore a kind of biomaterial, which are equipped with the function of cells but not contains cells' framework and maybe promising in clinical application. Based on the access to previous research, we hypothesized that hADSC-sEVs could be a kind of biomaterial applying in promoting vessels reconstruction after transplantation, with satisfactory biocompatibility and retention.

In this context, we extracted sEVs from the fourth generation of hADSCs and identified the characteristics of hADSCs and hADSC-sEVs. Then, we explore the mechanism of hADSC-sEVs' exerting influence in this biological progress. We chose a nude mice fat grafting model to indentify whether hADSC-sEVs could potentially promote angiogenesis after fat grafting and studied the underlying mechanism of hADSCsEVs' effect in improving the retention of fat graft.

\section{Materials And Methods}

\section{Animal maintenance}

All animal protocols were implemented under the Animal Ethical Committee of Fujian Medical University's supervision and approval. Eighteen male nude mice (6 weeks of age) were raised in the Experimental Animal Center of Fujian Medical University. Animals were kept in cages individually after wounding and maintained under ambient temperature.

\section{Cell culture}

Adipose tissues were obtained from healthy people undergoing liposuction surgeries with informed consent and were used for hADSCs isolation and fat transplantation in nude mice model. This study was approved by the Ethics Committee of Union hospital of Fujian Medicine University and performed following the principles described in the Declaration of Helsinki. hADSCs were isolated as previously described [15]. Briefly, the lipoaspirate was washed with phosphate-buffered saline (PBS) and digested with $0.25 \%$ collagenase I (Sigma, Aldrich, St. Louis, MO, USA). After filtration and centrifugation, the cell pellet was resuspended in Dulbecco's Modified Eagle Medium (DMEM)(Hyclone, USA) supplemented with $10 \%$ fetal bovine serum (FBS, Gibco, Carlsbad, CA, USA) and then cultured in an incubator with $37^{\circ} \mathrm{C}$ and $5 \% \mathrm{CO}_{2}$. The fourth generation of hADSCs was used in future experiments. The collected hADSCs was observed by inverted microscope (CNOPTEC, Chongqing, P. R. China) and characterized by osteogenic and adipogenic induction and flow cytometry (BD Biosciences, San Jose, CA, USA). 


\section{Identification of hADSCs}

We chose the fourth-generation hADSCs, which were in the state of logarithmic growth. When the cells grow to more than $80 \%-90 \%$ fusion, we added adipogenesis induction fluid (a mixture of $1 \mu \mathrm{mol} / \mathrm{L}$ dexamethasone, $10 \mu \mathrm{mol} / \mathrm{L}$ insulin, $200 \mu \mathrm{mol} / \mathrm{L}$ indomethacin, $0.5 \mathrm{mmol} / \mathrm{L}$ isobutylmethylxanthine and completely medium), and changed the medium every 3 days. Oil Red Assay kit (KeyGEN BioTECH, Jiangsu, China) was for lipid droplets staining according to the manufacturer's specifications, and the results were observed under a microscope after 2 weeks.

Similarly, we used the fourth-generation hADSCs which increased in logarithmically and added osteogenic induction fluid (a mixture of $10 \mathrm{mmol} / \mathrm{L} \beta$-glycerol sodium phosphate, $0.1 \mu \mathrm{mol} / \mathrm{L}$ dexamethasone, $50 \mu \mathrm{mol} / \mathrm{L}$ vitamin $\mathrm{C}$ and complete medium) after the cells grew to more than $80 \%-90 \%$ fusion. Accordingly, we changed the medium every 3 days and used alkaline phosphatase calcium cobalt staining kit (KeyGEN BioTECH, Jiangsu, China) for staining cells after 3 weeks according to the manufacturer's instructions and the results were observed under the microscope.

\section{Flow cytometry}

The fourth-generation of hADSCs was selected for flow cytometry analysis for phenotypic identification of mesenchymal stem cells. CD29, CD90, CD31 and CD45 along with related isotype controls (Abcam, Cambridge, UK) were used for hADSCs' staining. Flow cytometry was performed by using the BD Accuri C6 System (BD Biosciences, San Jose, CA, USA).

\section{Acquisition of hADSC-sEVs}

hADSC-sEVs were collected and purified according to the following processes. After cells' fusion rate reaching $70 \%-80 \%$, hADSCs' culture medium was replaced with serum-free low glucose DMEM for 48 hours to collect cells' supernatant. To isolate and remove cell particles, dead cells and cell debris of the obtained supernatant, we performed a series of differential centrifugal precipitation (300xg for $10 \mathrm{~min}$, $2,000 \times \mathrm{g}$ for $10 \mathrm{~min}$ and $10,000 \times \mathrm{g}$ for $30 \mathrm{~min}$ ). The supernatant removed the sediment was then filtered through a $0.22 \mu \mathrm{m}$ filter (Millipore, USA) to remove the large extracellular vesicles further and ultracentrifuged at $100,000 \times \mathrm{g}$ for 70 min by using the High-Speed Refrigerated Centrifuge (Beckman Coulter, USA). The supernatant was discarded, and the precipitation was resuspended with PBS. Finally, the suspension was ultracentrifuged at $100,000 \times \mathrm{g}$ for $70 \mathrm{~min}$ again, and sEVs were obtained after precipitation collection. The obtained sEVs concentration was measured with bicinchoninic acid (BCA) protein detection kit (Beyotime, Shanghai, China) and stored at $-80^{\circ} \mathrm{C}$ for further use. All centrifugations operated at $4^{\circ} \mathrm{C}$.

\section{Transmission electron microscopy}

EVs were imaged by transmission electron microscopy (TEM) to verify their morphology. The sample with a volume of $5 \mu \mathrm{l}(366 \mu \mathrm{g} / \mathrm{mL})$ was prepared and dropped on the sealing film. Covered with a copper mesh and stood for $20 \mathrm{~min}$ so that the copper mesh fully absorbed sEVs. The copper mesh with sEVs adsorbed 
was transferred to $4 \%$ paraformaldehyde for fixation for $5 \mathrm{~min}$. Then using $50 \mu \mathrm{l}$ of $2 \%$ uranyl acetate to stain negatively with copper mesh for $5 \mathrm{~min}$, and then copper mesh was dried at room temperature for 30 min. Finally, using FEl transmission electron microscopy $\mathbb{F E I}$ Tecnai G2, USA囚for imaging at $100 \mathrm{kV}$.

\section{Scanning electron microscopy}

Take $20 \mu \mathrm{l} \mathrm{sEVs} \mathrm{samples} \mathrm{and} \mathrm{freeze-dry} \mathrm{them} \mathrm{in} \mathrm{a} \mathrm{freeze} \mathrm{dryer} \mathrm{for} 16 \mathrm{~h}$. Then, the lyophilized sEVs powder was evenly dispersed on the conductive tape of the sample holder, and the sample holder was placed in the gold evaporation chamber for ion sputtering gold plating. Finally, the shape and quantity of sEVs were observed under a high-low vacuum scanning electron microscope (SEM)(FEI QUANTA 450, USA), and the images were taken and recorded.

\section{Nanoflow analysis}

Taking $30 \mu \mathrm{L}$ of sEVs diluent and adding FITC Mouse Anti-Human CD9, CD81 (BD, Franklin Lake, New Jersey, USA) and FITC Mouse IgG (BioLegend, San Diego, USA) to mix, then incubating at $37^{\circ} \mathrm{C}$ for $30 \mathrm{~min}$ in the dark, adding $1 \mathrm{~mL}$ of pre-cooled PBS, 110,000 $\times$ g ultracentrifuging for $70 \mathrm{~min}$. Carefully remove the supernatant, resuspend it in $50 \mu \mathrm{L}$ of pre-cooled PBS, and detect the protein index results with a NanoFCM instrument (NanoFCM, China).

\section{Nanoparticle tracking analysis}

Particle size, particle size distribution and concentration of sEVs were identified by nanoparticle tracking analysis (NTA)(NanoFCM, China). Compared with polystyrene beads (RI=1.59), in the Nano-FCM system, monodisperse silica nanoparticles $(\mathrm{Rl}=1.46)$ are employed as the reference to calibrate the size of $\mathrm{EVs}$. In the nanoFCM system, the detection efficiency is $100 \%$. Particle concentration can be determined via single-particle enumeration, which defines the particle concentration of the number of particles collected in a given period. Finally, the size, distribution and total concentration of EVs were calculated by NTA software.

\section{Zeta potential assay of sEVs}

Zeta potentials of $s E V s$ were measured three times using a Nano laser particle size analyzer (Litesizer 500, Anton Paar, Austria). Data were collected and analyzed using Anton Paar Kalliope software.

\section{Internalization of hADSC-sEVs into HUVECs}

The hADSC-sEVs were labelled with a PKH67 dye (Sigma Aldrich, USA) for 4min. Then, Bovine Serum Albumin (BSA) was used to terminate staining. The excess dye after labelling was removed by ultracentrifugation at 100,000 $\mathrm{g}$ for $1 \mathrm{~h}$ at 4 degrees. HUVECs (Cellcook, Guangzhou, China) and hADSCsEVs labelled with PKH67 were incubated in $37^{\circ} \mathrm{C}$ with serum-free medium for $6 \mathrm{~h}$ and $12 \mathrm{~h}$. After fixation with $4 \%$ paraformaldehyde (PFA) for 30min and staining with 4, 6-diamino-2-phenylindoles (DAPI, CST, USA), the sample was observed under a fluorescence microscope (Nikon, Japan). 


\section{Histological analysis \& immunostaining}

The grafted fat paraffin-embedded sections were stained by immunohistochemistry. The sections were primarily incubated with anti-CD34 antibody, anti-VEGFR2, anti-Ki-67 (Abcam, USA), followed by incubation with horseradish peroxidase-conjugated secondary antibody. Finally, the staining colour was developed using the DAB Detection Kit (Maixin, Fuzhou, China).

\section{Animal studies}

Six-week-old, male nude mice were obtained from the Laboratory Animal Center of Fujian medicine University (Fuzhou, P. R. China). The experimental protocol was approved by the Animal Care and Use Committee of our institution. The mice were randomly assigned to hADSC-sEVs or control groups (six mice in each group). A mixture of $0.4 \mathrm{ml}$ of aspirated fat and $0.1 \mathrm{ml}\left(10^{10}\right.$ particles/ml) hADSC-sEVs solution (hADSC-sEVs group) or $0.1 \mathrm{ml}$ PBS (control group) was injected subcutaneously into the back of nude mice. The mice were sacrificed at 1, 2 and 3 months after fat grafting. Grafted fat samples were harvested and measured by the weight, volume, hematoxylin-eosin (HE) and immunochemistry staining, which included an assessment of fat graft integrity, as evidenced by the presence of intact and nucleated adipocytes; the presence of cysts and vacuoles. Each parameter was graded by two observers independently on a semiquantitative scale ranging from 0 to $5(0=$ absence; $1=$ minimal presence; $2=$ minimal to moderate presence; $3=$ moderate presence; $4=$ moderate to extensive presence; and $5=$ extensive presence).

\section{Statistical analysis}

Statistical analysis was performed using GraphPad Prism 8.0.1 software (GraphPad Software, Inc., La Jolla, CA, USA). Results were presented as the mean \pm standard deviation (SD). The two-tailed Student's ttest was used to evaluate differences between groups. $\mathrm{P}<0.05$ was considered as statistically significant.

\section{Results}

\section{Characterizations of ADSCs}

Inverted fluorescence microscope revealed a characteristic morphology of slender spindle-like cells of hADSCs (Figure 1A). Mature adipocytes and mineralized nodules can be formed from hADSCs induced by adipogenic and osteoblastic induction fluid, respectively (Figure 1B, C). The results of oil red $\mathrm{O}$ staining showed that there were red fat droplets with different sizes in the cells, and the alkaline phosphatase calcium cobalt method staining showed positive osteoblastic nodules. To determine the mesenchymal phenotype of hADSCs, we investigated the purified hADSCs by using flow cytometry analysis. Approximately $98 \%$ of hADSCs were positive for CD29 and CD90, but negative for CD31 and CD45 (Figure 1D). These suggested that the cultured cells were adipose-derived stem cells [17]. 


\section{Characterizations of hADSC-sEVs}

In order to obtain hADSC-sEVs, we used the classic ultracentrifugation method. The morphology of sEVs was observed under transmission electron microscopy. It was a saucer-like membrane structure with a diameter of $50 \sim 120 \mathrm{~nm}$ (Figure 2A). Under the scanning electron microscopy, the sEVs can be seen in a more objective and accurate spherical three-dimensional shape, with particle size similar to that of the transmission electron microscope (Figure 2B). Specific markers CD9 and CD81 of EVs were confirmed to be expressed in hADSC-sEVs, and IgG was used as a negative control (Figure 2C). According to the results of NTA data analysis, most of the sEVs'particle size ranged between 50-120 nm (Figure 2D). Besides, Zeta potential measurement of sEVs showed that its average potential was $-16.68 \mathrm{mV}$ (Figure 2E).

\section{hADSC-sEVs can be transferred to human umbilical vein endothelial cells}

As shown in Figure 3, the PKH67-labeled hADSC-sEVs were co-incubated with human umbilical vein endothelial cells for $6 \mathrm{~h}$ and $12 \mathrm{~h}$. The green fluorescence spots were scattered around the nucleus of HUVECs. These results indicate that the hADSC-sEVs were increasingly distributed around the core as time went on. Which means hADSC-sEVs play its role mainly through internalization into cells.

\section{hADSC-sEVs improved the fat graft survival rate in the nude mice model}

To assess the pro-angiogenic potential of hADSC-sEVs, we adopted a nude mice model of fat grafting. The grafts were harvested 1, 2 and 3 months after fat transplantation. Gross observation of the graft specimens demonstrated that the hADSC-sEVs group had larger graft sizes compared to that in the control group (Figure 4A). This was confirmed by the weight and volume measurements of the grafts $(P<$ 0.05). A significantly higher graft survival rate was observed in the hADSC-sEVs group when compared with the control group from 1 to 8 weeks after fat grafting. As shown in Figure 4B and 4C, 1, 2 and 3 months after fat grafting, the weight and volume of grafted fat were more significant in the hADSC-sEVs group compared to the control group $(P<0.05)$, indicating a protective effect of hADSC-sEVs on grafted fat survival.

\section{hADSC-sEVs promoted neovascularisation in the nude mice model of fat grafting}

We used HE and Immunohistologic staining for histological evaluation. HE staining revealed that the grafted fat in the hADSC-sEVs groups exhibited better survival and morphologic integrity compared to the control group, as shown in Figure 5A. We observed extensive cystic changes and fibrous septa in the control group. There were significant differences between the hADSC-sEVs groups and control group in the histological evaluation of integrity, cysts/vacuoles, fibrosis, and inflammation $(P<0.05)$ (Figure 5B, C). Studies have shown that vascularisation is crucial for fat survival and regeneration [18]. So we measured capillary density within the grafts via immunohistochemical (IHC) staining of anti-CD34 antibody in tissue sections to evaluate the effect of hADSC-sEVs on neoangiogenesis of grafted fat. Consistent with the HE staining and histologic examination, IHC staining showed a significant increase in CD34-positive (a specific marker of capillary density [19]) in the hADSC-sEVs group with the control 
groups (Figure 6), indicating increased capillary density in the hADSC-sEVs group. Together, these results showed that hADSC-sEVs could effectively improve the vascularisation of the grafted fat.

\section{Mechanism of hADSC-sEVs-mediated Angiogenesis in vivo}

The previous study has revealed that VEGF secreted by stromal cells stimulates the proliferation and survival of endothelial cells leading to the formation of new blood vessels [7]. We further evaluated the potential mechanism of hADSC-sEVs in promoting survival of grafted fat by IHC analysis of anti-VEGFR2. The results demonstrated that the expression of VEGFR2 was increased in the hADSC-sEVs group compared to the control group in 1, 2 and 3 months after transplantation (Figure 6), suggesting that VEGF were involved in hADSC-sEVs-mediated angiogenesis. We also semi-quantified cell proliferation by analyzing Ki-67 staining [20] and we found increased immunostaining of Ki-67 in the hADSC-sEVs group compared with the control group (Figure 6), suggesting that the proliferation of vascular endothelial cells were enhanced after hADSC-sEVs treatment.

\section{Discussion}

The study of EVs spans decades. In recent years, it has been brought to the fore, which is mainly due to the remarkable advancement of the identification of EVs and related mechanisms have been continuously explored [21]. In particular, hADSC-sEVs have shown great potential in a variety of disease models [22-25].

In this study, we used a classic ultracentrifugation method to separate the target $s E V s$, which obtained $s E V s$ with higher purity. After acquiring sEVs, we combined transmission electron microscopy and scanning electron microscopy to study the morphology, protein composition and physical property of hADSC-sEVs. The results of two-dimensional and three-dimensional images show that the sEVs we obtained were spherical double-layer membrane structure with a particle size range of about 50-150 nm, which was similar to other related literature [26]. Most of those vesicles (98\%) were 50-120 nm based on NTA analysis, and the mean size was $77 \mathrm{~nm}$, which was consistent with the acknowledged size range ( 30-150 nm ) [27]. The above showed that the classic ultracentrifugation method could be used to enrich nano-level EVs at a higher purity. Among membrane proteins, we used CD9 and CD81 as positive markers, and IgG as the negative control. CD9 and CD81 are usually associated with EVs and are often regarded as surface protein markers for EVs [28]. Through nanoflow analysis, we successfully detected the sEVs marker proteins CD9 (3.0\%), CD81 (10.1\%), and IgG (0.2\%). In terms of physical properties, hADSC-sEVs displayed negative zeta potential values. All these data indicated that the extracellular vesicles of hADSCs we extracted are small EVs, which is the operational term referred to EVs' size, actually [21].

The relationship between sEVs and vascular regeneration is the focus of research in the field of regenerative medicine. Studies have shown that $\mathrm{SEV}$ s can change the angiogenesis steps including proliferation, migration, and endothelial cell structure, as well as increase the expression of angiogenesisrelated genes and the secretion of related proteins, including VEGFA, CXCL8, IL-6, FGF2, miRNA-23a [2932]. To test whether $s E V s$ can improve the survival of grafted fat by promoting angiogenesis, we adopted 
the nude mice fat grafting model. Consistent with previous studies [29,33], we demonstrated that fat grafts in the hADSC-sEVs groups exhibited better survival and morphologic integrity compared to the control group.

Several factors are contributing to fat graft survival [9]. Since the grafted fat show lower tolerance for ischemia caused by devascularisation, it is quickly absorbed and replaced by fibrous tissues and oil sacs $[34,35]$. The development of a neovascular supply, or angiogenesis, serves crucial homeostatic roles since blood vessels carry nutrients to tissues and organs and remove catabolic products [36]. Therefore, timely and adequate neoangiogenesis is essential for the survival of grafted fat $[37,38]$. Substantial evidence demonstrates CD34 is expressed not only by MSC but by a multitude of other nonhematopoietic cell types, including vascular endothelial progenitors [39]. Therefore, we adopted CD34 as a maker of neovascularisation analysis. Our results showed a significant increase in CD34-positive in the hADSCsEVs group with the control groups (Figure 6), indicating increased capillary density in the hADSC-sEVs group. We can observe the improvement of angiogenesis in the hADSC-sEVs group, and then we began to focus on the underlying mechanism of those improvements. Studies have revealed that VEGF is identified as a principal pro-angiogenic factor that enhances the production of new blood vessels from the existing vascular network [40]. It is well established that VEGF/VEGFR2 signalling pathway plays a vital role in regulating the process of neoangiogenesis. And it was demonstrated that the lower-affinity, highly homologous VEGFR2 was the primary signalling receptor for VEGF [41]. So we chose VEGFR2 as our reserch target. Our IHC analysis demonstrated elevated VEGFR2 expression in grafted tissue of the hADSC-sEVs group. Therefore, the high expression of VEGFR2 in the hADSC-sEVs group may account for its pro-angiogenic effects. For further verification, we analyzed the $\mathrm{Ki}-67 \mathrm{IHC}$ staining and found increased immunostaining of Ki-67 in the hADSC-sEVs group compared with the control group, suggesting that hADSC-sEVs treatment could enhance the proliferation of vascular endothelial cells.

As a potential type of nanomaterial, sEVs has attracted growing attention from researchers in different fields. Interdisciplinary integration and the use of nanotechnology continuously promote the development of sEVs. However, in the process of extraction, identification and subsequent mechanism research of sEVs, we found that a large amount of supernatant was needed to extract only a little bit of sEVs (Figure 7). Therefore, how to obtain a large number of sEVs has become a critical preclinical and clinical research direction. Only by breaking the bottleneck of $s E V s$ production can the clinical transformation and application of sEVs have infinite development possibilities. We think that there are two main ways to increase the output of $s E V s$. One is to increase the number of $s E V s$ secreted by cells from the source, which is, microcarrier-based three-dimensional (3D) cell culture technology. The other is to reduce the loss of $s E V s$, which means in the process of extracting EVs, it is necessary not only to ensure its purity but also to minimize the loss of $s E V s$.

3D cell culture technology is a common strategy for large-scale adherent cell culture, which adopt a kind of device equipped with three-dimensional culture system based on a hollow fiber bioreactor, and a large amount of conditioned medium (CM) can be obtained by using this device. Studies have shown that compared with the traditional 2D culture, the total amount of sEVs in the 3D culture system has increased 
19.4 times [42,43]. Moreover, compared with 2D-sEVs, 3D-sEVs has no significant differences in surface markers, size, and shape. In particular, 3D-sEVs can significantly improve the symptoms of related diseases in animal models and are more effective than 2D-sEVs [44]. In conclusion, sEVs obtained by 3D cell culture are more in line with the needs of the body's biological functions, which is an important measure for the clinical development of sEVs.

In terms of $s E V s$ extraction, the mainstream $s E V s$ extraction methods mainly include ultracentrifugation (UC), density gradient centrifugation (DGC), exclusion chromatography (SEC), ultrafiltration (UF), Immune capture (IC) and polymer precipitation (Precip) [45]. Among them, the most classic method is UC [46], but its main limitations are time-consuming, costly and low yield. So people began the exploration of how to increase the output of $s E V s$ further and reduce the cost while ensuring its purity. In recent years, preclinical studies have found that the extraction method of UF combined with SEC is superior to the ultracentrifugation method under the comprehensive conditions of purity, efficiency and cost [47-50]. In addition, in industrial production, mass production of high-quality sEVs is the most critical factor in its therapeutic applications. Among various separation methods, tangential flow filtration (TFF) is considered as an ideal method for industrial-scale production of sEVs [51-53]. TFF can provide GMP level sEVs from a large amount of CM [54]. Some studies have even shown that sEVs separated by TFF have higher yield and activity than those separated by ultracentrifugation [42,55]. And the analysis of multiple batches of isolated MSC-sEVs showed that the TFF method could generate stable sEVs in a large volume of media. Therefore, TFF is suitable for large-scale production of high-quality sEVs that meet GMP requirements [44].

The present study had several limitations. First, the downstream molecules in the VEGF/VEGFR2 signalling pathway remain to be defined in our further investigation. Second, the theory of graft retention or endogenous adipose regeneration is still under defing. Consequently, further studies are warranted to address this issue.

\section{Conclusion}

Small extracellular vesicles, as a novel kind of nanoparticle without nuclear structure, do not show apparent side effects, such as immunogenicity or tumorigenicity when applied in animal models. Studies have found that EVs can replicate the function of the cells which they are derived. Our research has proved that hADSC-SEVs play a considerable role in fat grafting nude mice model. hADSC-sEVs can promote neovascularization and increase the retention of grafted fat, whose mechanism may be explained by VEGF/VEGFR2 signal transduction. These findings indicate that hADSC-sEVs can be regarded as a potential treatment option for fat transplantation. As a new type of nanomaterial, we need further and more in-depth studies to promote hADSC-sEVs to apply in a broader range of diseases.

\section{Abbreviations}


ADSCs: adipose-derived stem cells; DMEM: Dulbecco's modified Eagle's medium; PBS: phosphatebuffered

saline; sEVs: Small extracellular vesicles; hADSC-sEVs:the small extracellular vesicles of human adiposederived stem cells; HUVECs: human umbilical vein endothelial cells; MSCs: mesenchymal stem/stromal cells; SVF:stromal vascular fraction; CAL: cell-assisted lipotransfer; FBS:fetal bovine serum; BCA:

bicinchoninic acid; TEM: transmission electron microscopy; SEM:scanning electron microscope; NTA: nanoparticle tracking analysis; BSA: Bovine Serum Albumin; PFA: paraformaldehyde; DAPI: 4, 6-diamino2-phenylindoles; HE: hematoxylin-eosin; SD: standard deviation; CM: conditioned medium; UC: ultracentrifugation; DGC: density gradient centrifugation; SEC:exclusion chromatography; UF: ultrafiltration; IC: Immune capture; Precip: polymer precipitation; TFF: tangential flow filtration.

\section{Declarations}

\section{Ethics approval and consent to participate}

All animal protocols were implemented under the Animal Ethical Committee of Fujian Medical University's (PR China) supervision and approval, which conforms to the guide for the National Institutes of Health to use laboratory animals

\section{Consent for publication}

Not applicable.

\section{Availability of data and materials}

The data that support the findings of this study are available from the corresponding author upon reasonable request.

\section{Competing interests}

The authors have declared that no competing interest exists.

\section{Funding}

This study was financed by National Natural Science Foundation of China (grant No.81971855, 81671930), Joint Funding Project of Science and Technology Innovation in Fujian Province (grant No. 2017Y9101), Special Financial Funds of Fujian Province (grant No. 2018B054).

\section{Authors' contributions}

Conceptualization: AZC and SJT designed, performed experiments, analyzed data, and wrote manuscript. JWH and XYL performed experiments. JHC and GHP edited manuscript. LWC and HRZ conceived, designed experiments, and reviewed/edited manuscript. XSC is the guarantor of this work and, as such, 
had full access to all the data in this study and takes responsibility for the integrity of the data and the accuracy of the data analysis. All authors have read and agreed to the published version of the manuscript.

\section{Acknowledgements}

We thank the Public Technology Service Center of Fujian Medical University (PR China) and Central Laboratory of Fujian Medical University Union Hospital (PR China) for providing technical support as well as experimental platforms. We appreciate Xuejing Zeng, Deye Zeng's (Fujian Medical University Union Hospital, PR China) assistance for the interpretation, and preparation of the histological section.

\section{References}

1. Vidal M. Exosomes and GPI-anchored proteins: Judicious pairs for investigating biomarkers from body fluids. Advanced drug delivery reviews. 2020.

2. Borrelli DA, Yankson K, Shukla N, Vilanilam G, Ticer T, Wolfram J. Extracellular vesicle therapeutics for liver disease. Journal of controlled release : official journal of the Controlled Release Society. 2018;273:86-98.

3. van Balkom BWM, Gremmels H, Giebel B, Lim SK. Proteomic Signature of Mesenchymal Stromal Cell-Derived Small Extracellular Vesicles. Proteomics. 2019;19(1-2):e1800163.

4. Cui X, He Z, Liang Z, Chen Z, Wang H, Zhang J. Exosomes From Adipose-derived Mesenchymal Stem Cells Protect the Myocardium Against Ischemia/Reperfusion Injury Through Wnt/ $\beta$-Catenin Signaling Pathway. J Cardiovasc Pharmacol. 2017;70(4):225-31.

5. Hu L, Wang J, Zhou X, Xiong Z, Zhao J, Yu R, et al. Exosomes derived from human adipose mensenchymal stem cells accelerates cutaneous wound healing via optimizing the characteristics of fibroblasts. Sci Rep. 2016;6:32993.

6. Merino-Gonzalez C, Zuniga FA, Escudero C, Ormazabal V, Reyes C, Nova-Lamperti E, et al. Mesenchymal Stem Cell-Derived Extracellular Vesicles Promote Angiogenesis: Potencial Clinical Application. Front Physiol. 2016;7:24.

7. Liu X, Li Q, Niu X, Hu B, Chen S, Song W, et al. Exosomes Secreted from Human-Induced Pluripotent Stem Cell-Derived Mesenchymal Stem Cells Prevent Osteonecrosis of the Femoral Head by Promoting angiogenesis. Int J Biol Sci. 2017;13(2):232-44.

8. Basu J, Ludlow JW. Exosomes for repair, regeneration and rejuvenation. Expert Opin Biol Ther. 2016;16(4):489-506.

9. Yu Q, Cai Y, Huang H, Wang Z, Xu P, Wang X, et al. Co-Transplantation of Nanofat Enhances Neovascularization and Fat Graft Survival in Nude Mice. Aesthet Surg J. 2018;38(6):667-75.

10. Bellini E, Grieco MP, Raposio E. The science behind autologous fat grafting. Ann Med Surg (Lond). 2017;24:65-73. 
11. Konczalik W, Siemionow M. Experimental and clinical methods used for fat volume maintenance after autologous fat grafting. Ann Plast Surg. 2014;72(4):475-83.

12. Yoshimura K, Sato K, Aoi N, Kurita M, Hirohi T, Harii K. Cell-Assisted Lipotransfer for Cosmetic Breast Augmentation: Supportive Use of Adipose-Derived Stem/Stromal Cells. Aesthetic Plast Surg. 2020.

13. Suga $H$, Glotzbach JP, Sorkin M, Longaker MT, Gurtner GC. Paracrine mechanism of angiogenesis in adipose-derived stem cell transplantation. Ann Plast Surg. 2014;72(2):234-41.

14. Bacakova L, Zarubova J, Travnickova M, Musilkova J, Pajorova J, Slepicka P, et al. Stem cells: their source, potency and use in regenerative therapies with focus on adipose-derived stem cells - a review. Biotechnology advances. 2018;36(4):1111-26.

15. Laloze J, Varin A, Gilhodes J, Bertheuil N, Grolleau JL, Brie J, et al. Cell-assisted lipotransfer: Friend or foe in fat grafting? Systematic review and meta-analysis. J Tissue Eng Regen Med. 2018;12(2):e1237-e50.

16. Eun SC. Stem cell and research in plastic surgery. J Korean Med Sci. 2014;29 Suppl 3:S167-9.

17. Wang D, Liu N, Xie Y, Song B, Kong S, Sun X. Different culture method changing CD105 expression in amniotic fluid MSCs without affecting differentiation ability or immune function. $J$ Cell Mol Med. 2020; 24(7):4212-22.

18. Zheng $H, Y u Z$, Deng $M$, Cai $Y$, Wang $X, X u Y$, et al. Fat extract improves fat graft survival via proangiogenic, anti-apoptotic and pro-proliferative activities. Stem Cell Res Ther. 2019;10(1):174.

19. Xue Y, Lim S, Brakenhielm E, Cao Y. Adipose angiogenesis: quantitative methods to study microvessel growth, regression and remodeling in vivo. Nat Protoc. 2010;5(5):912-20.

20. Rotti PG, Xie W, Poudel A, Yi Y, Sun X, Tyler SR, et al. Pancreatic and Islet Remodeling in Cystic Fibrosis Transmembrane Conductance Regulator (CFTR) Knockout Ferrets. Am J Pathol. 2018;188(4):876-90.

21. Thery C, Witwer KW, Aikawa E, Alcaraz MJ, Anderson JD, Andriantsitohaina R, et al. Minimal information for studies of extracellular vesicles 2018 (MISEV2018): a position statement of the International Society for Extracellular Vesicles and update of the MISEV2014 guidelines. J Extracell Vesicles. 2018;7(1):1535750.

22. Kholia S, Ranghino A, Garnieri P, Lopatina T, Deregibus MC, Rispoli P, et al. Extracellular vesicles as new players in angiogenesis. Vascul Pharmacol. 2016;86:64-70.

23. Ribeiro-Rodrigues TM, Laundos TL, Pereira-Carvalho R, Batista-Almeida D, Pereira R, Coelho-Santos V, et al. Exosomes secreted by cardiomyocytes subjected to ischaemia promote cardiac angiogenesis. Cardiovasc Res. 2017;113(11):1338-50.

24. Zhang B, Wu X, Zhang X, Sun Y, Yan Y, Shi H, et al. Human umbilical cord mesenchymal stem cell exosomes enhance angiogenesis through the Wnt4/beta-catenin pathway. Stem Cells Transl Med. 2015;4(5):513-22.

25. Wong DE, Banyard DA, Santos PJF, Sayadi LR, Evans GRD, Widgerow AD. Adipose-derived stem cell extracellular vesicles: A systematic review(). J Plast Reconstr Aesthet Surg. 2019;72(7):1207-18. 
26. Kondratov KA, Petrova TA, Mikhailovskii VY, Ivanova AN, Kostareva AA, Fedorov AV. EXTRACELLULAR VESICLES FROM BLOOD PLASMA STUDIED BY LOW VOLTAGE SCANNING ELECTRON MICROSCOPY. Tsitologiia. 2017.

27. Boukouris $S$, Mathivanan $S$. Exosomes in bodily fluids are a highly stable resource of disease biomarkers. Proteomics Clin Appl. 2015;9(3-4):358-67.

28. Raposo G, Stoorvogel W. Extracellular vesicles: exosomes, microvesicles, and friends. J Cell Biol. 2013;200(4):373-83.

29. Han Y, Ren J, Bai Y, Pei X, Han Y. Exosomes from hypoxia-treated human adipose-derived mesenchymal stem cells enhance Angiogenesis through VEGF/VEGF-R. Int J Biochem Cell Biol. 2019;109:59-68.

30. Montemurro T, Vigano M, Ragni E, Barilani M, Parazzi V, Boldrin V, et al. Angiogenic and antiinflammatory properties of mesenchymal stem cells from cord blood: soluble factors and extracellular vesicles for cell regeneration. Eur J Cell Biol. 2016;95(6-7):228-38.

31. McBride JD, Rodriguez-Menocal L, Guzman W, Candanedo A, Garcia-Contreras M, Badiavas EV. Bone Marrow Mesenchymal Stem Cell-Derived CD63 Exosomes Transport Wnt3a Exteriorly and Enhance Dermal Fibroblast Proliferation, Migration, and Angiogenesis In Vitro. Stem Cells Dev. 2017.

32. Mathiyalagan P, Liang Y, Kim D, Misener S, Thorne T, Kamide CE, et al. Angiogenic Mechanisms of Human CD34 Stem Cell Exosomes in the Repair of Ischemic Hindlimb. Circ Res. 2017.

33. Cui X HZ, Liang Z, et al. Exosomes From Adipose-derived Mesenchymal Stem Cells Protect the Myocardium Against Ischemia/Reperfusion Injury Through Wnt/b-Catenin Signaling Pathway. J Cardiovasc Pharmacol. 2017;70:225-231.

34. Dong Z, Peng Z, Chang Q, Zhan W, Zeng Z, Zhang S, et al. The angiogenic and adipogenic modes of adipose tissue after free fat grafting. Plast Reconstr Surg. 2015;135(3):556e-67e.

35. Mizoguchi T, Kijima Y, Hirata M, Kaneko K, Arima H, Nakajo A, et al. Histological findings of an autologous dermal fat graft implanted onto the pectoralis major muscle of a rat model. Breast Cancer. 2015;22(6):578-85.

36. Apte RS, Chen DS, Ferrara N. VEGF in Signaling and Disease: Beyond Discovery and Development. Cell. 2019;176(6):1248-64.

37. Locke MB dCT. Current practice in autologous fat transplantation: suggested clinical guidelines based on a review of recent literature. Ann Plast Surg. 2008;60: 98-102.

38. Yamaguchi M MF, Bujo $\mathrm{H}$, et al. Revascularisation determines volume retention and gene expression by fat grafts in mice. Exp Biol Med (Maywood). 2005;230: 742-748.

39. Sidney LE, Branch MJ, Dunphy SE, Dua HS, Hopkinson A. Concise review: evidence for CD34 as a common marker for diverse progenitors. Stem cells (Dayton, Ohio). 2014;32(6):1380-9.

40. Alkharsah KR. VEGF Upregulation in Viral Infections and Its Possible Therapeutic Implications. International journal of molecular sciences. 2018;19(6). 
41. Abhinand CS, Raju R, Soumya SJ, Arya PS, Sudhakaran PR. VEGF-A/VEGFR2 signaling network in endothelial cells relevant to angiogenesis. J Cell Commun Signal. 2016;10(4):347-54.

42. Haraszti RA, Miller R, Stoppato M, Sere YY, Coles A, Didiot MC, et al. Exosomes Produced from 3D Cultures of MSCs by Tangential Flow Filtration Show Higher Yield and Improved Activity. Mol Ther. 2018;26(12):2838-47.

43. Cao J, Wang B, Tang T, Lv L, Ding Z, Li Z, et al. Three-dimensional culture of MSCs produces exosomes with improved yield and enhanced therapeutic efficacy for cisplatin-induced acute kidney injury. Stem Cell Res Ther. 2020;11(1):206.

44. Yan L, Wu X. Exosomes produced from 3D cultures of umbilical cord mesenchymal stem cells in a hollow-fiber bioreactor show improved osteochondral regeneration activity. Cell Biol Toxicol. 2020;36(2):165-78.

45. Coumans FAW, Brisson AR, Buzas El, Dignat-George F, Drees EEE, El-Andaloussi S, et al. Methodological Guidelines to Study Extracellular Vesicles. Circ Res. 2017;120(10):1632-48.

46. Park K-S, Bandeira E, Shelke GV, Lässer C, Lötvall J. Enhancement of therapeutic potential of mesenchymal stem cell-derived extracellular vesicles. Stem Cell Res Ther. 2019.

47. Takov K, Yellon DM, Davidson SM. Comparison of small extracellular vesicles isolated from plasma by ultracentrifugation or size-exclusion chromatography: yield, purity and functional potential. $J$ Extracell Vesicles. 2019;8(1):1560809.

48. Corso G, Mäger I, Lee Y, Görgens A, Bultema J, Giebel B, et al. Reproducible and scalable purification of extracellular vesicles using combined bind-elute and size exclusion chromatography. Sci Rep. 2017;7(1):11561.

49. Buschmann D, Kirchner B, Hermann S, Märte M, Wurmser C, Brandes F, et al. Evaluation of serum extracellular vesicle isolation methods for profiling miRNAs by next-generation sequencing. $\mathrm{J}$ Extracell Vesicles. 2018;7(1):1481321.

50. Mol EA, Goumans MJ, Doevendans PA, Sluijter JPG, Vader P. Higher functionality of extracellular vesicles isolated using size-exclusion chromatography compared to ultracentrifugation. Nanomedicine. 2017;13(6):2061-5.

51. Reiner AT, Witwer KW, van Balkom BWM, de Beer J, Brodie C, Corteling RL, et al. Concise Review: Developing Best-Practice Models for the Therapeutic Use of Extracellular Vesicles. Stem Cells Transl Med. 2017.

52. Ha DH, Kim H-K, Lee J, Kwon HH, Park G-H, Yang SH, et al. Mesenchymal Stem/Stromal Cell-Derived Exosomes for Immunomodulatory Therapeutics and Skin Regeneration. Cells. 2020;9(5):1157.

53. Dizon-Maspat J, Bourret J, D'Agostini A, Li F. Single pass tangential flow filtration to debottleneck downstream processing for therapeutic antibody production. Biotechnol Bioeng. 2012;109(4):962-70.

54. Lee JH, Ha DH, Go HK, Youn J, Kim HK, Jin RC, et al. Reproducible Large-Scale Isolation of Exosomes from Adipose Tissue-Derived Mesenchymal Stem/Stromal Cells and Their Application in Acute Kidney Injury. Int J Mol Sci. 2020;21(13). 
55. Busatto S, Vilanilam G, Ticer T, Lin W-L, Dickson DW, Shapiro S, et al. Tangential Flow Filtration for Highly Efficient Concentration of Extracellular Vesicles from Large Volumes of Fluid. Cells. 2018;7(12):273.

\section{Figures}

A

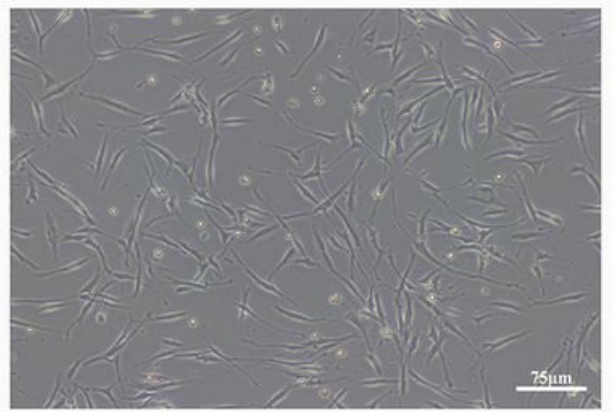

B
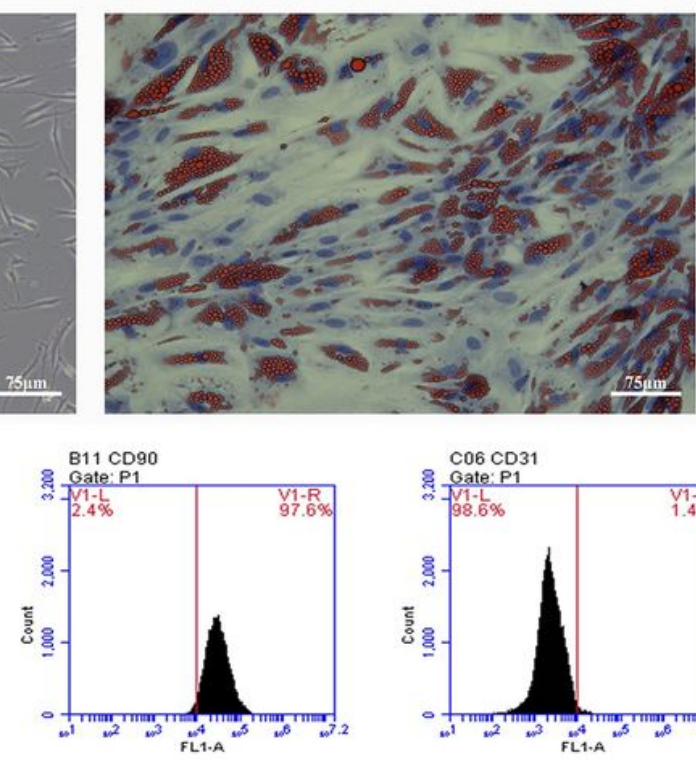

D
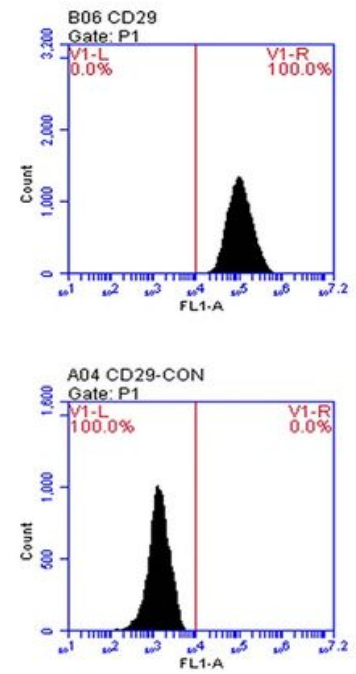

A07 CD90-CON

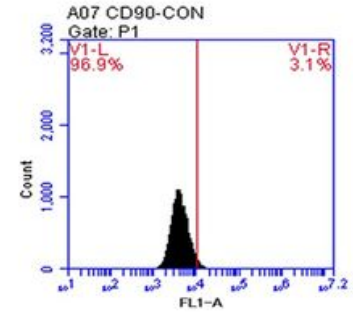

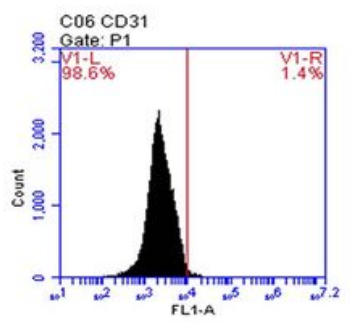
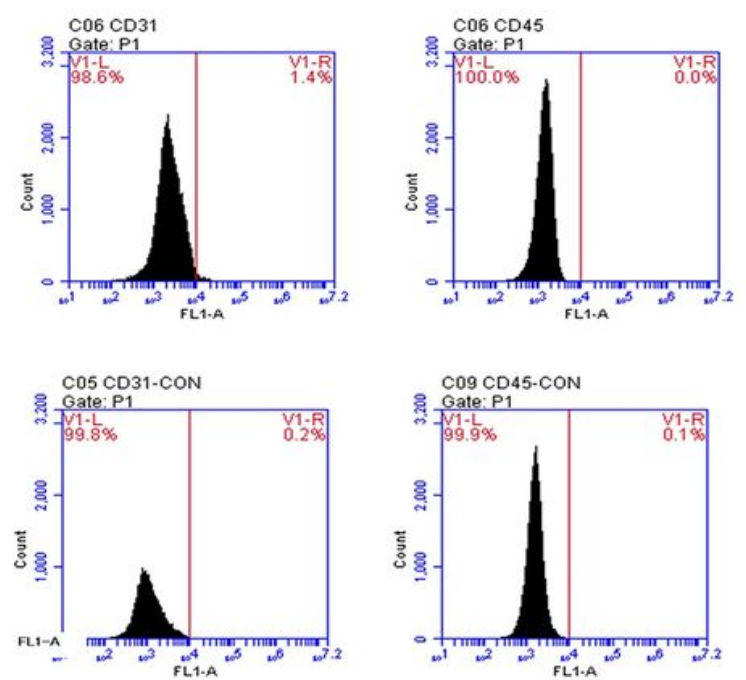

C

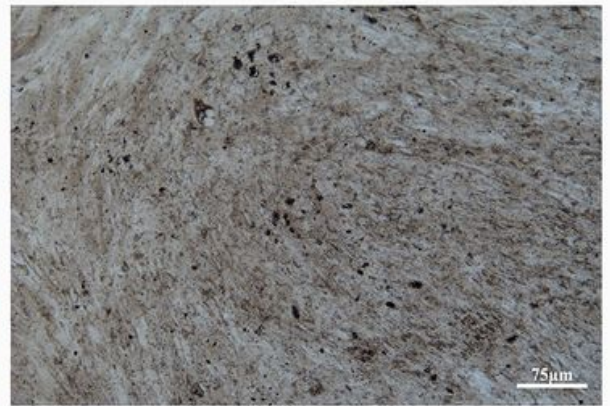

\section{Figure 1}

Characterizations of human adipose-derived stem cells (hADSCs). (A) Morphological features of the fourth-generation hADSCs under light microscopy (scale bar $=75 \mathrm{~nm}$ ). (B) Representative images of Oil Red $O$ staining. (scale bar $=75 \mathrm{~nm}$ ). (C) the alkaline phosphatase calcium cobalt staining showed positive osteoblastic nodules (scale bar $=75 \mathrm{~nm}$ ). (D) Flow cytometry analysis of cell surface expression of CD29, CD90, CD31 and CD45 proteins in hADSCs. 
A

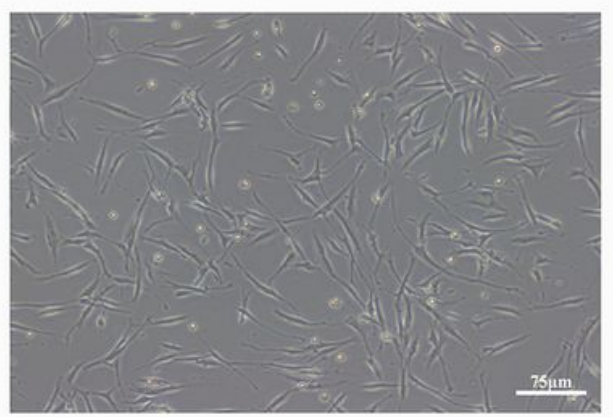

B

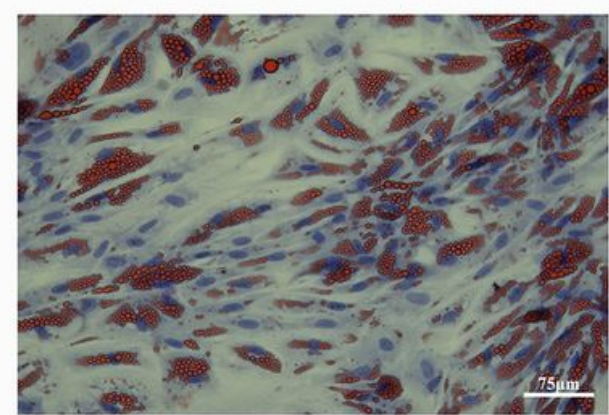

D
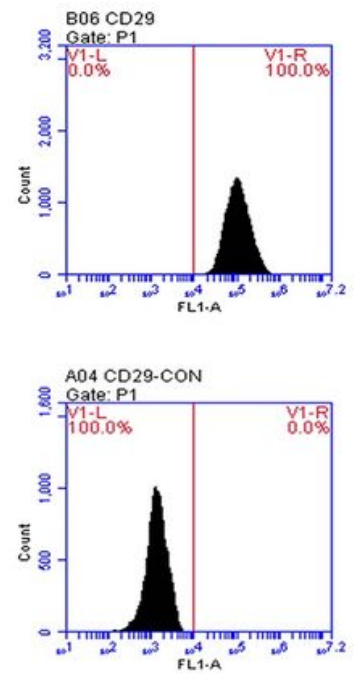
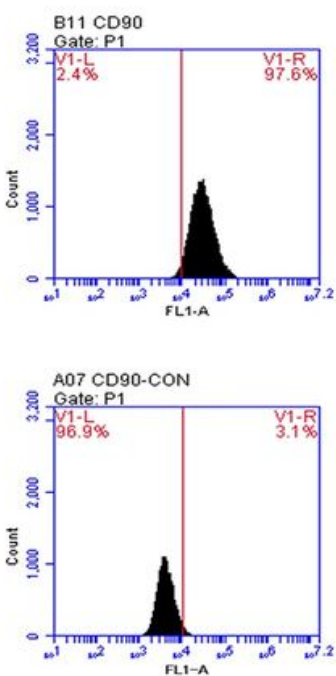
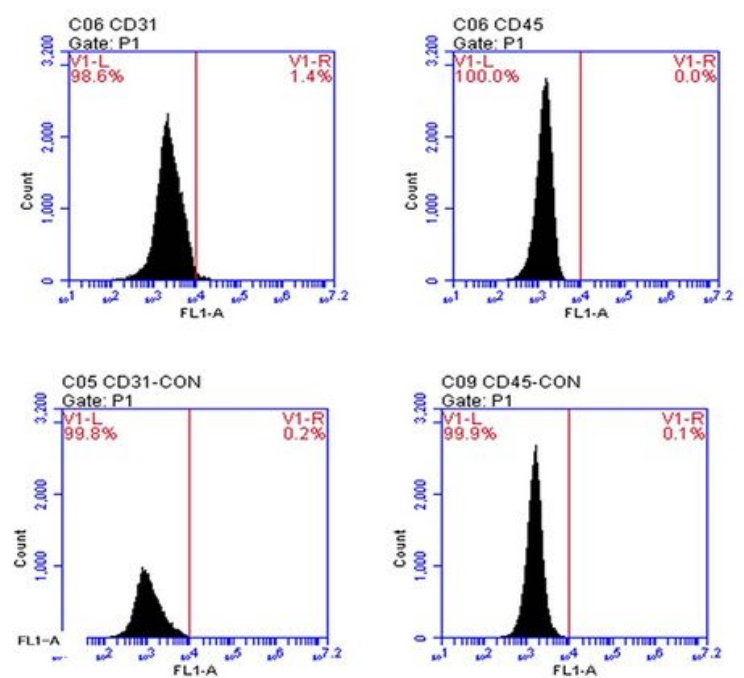

C

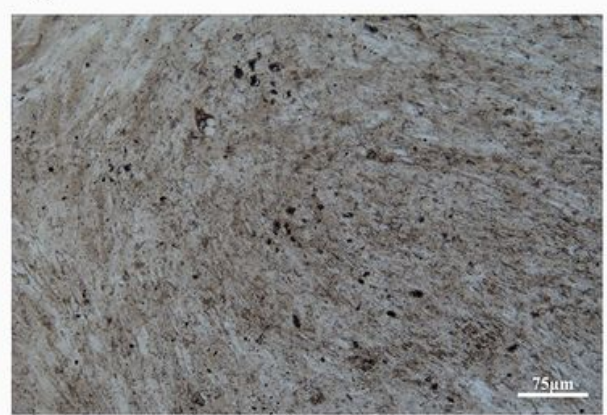

Figure 1

Characterizations of human adipose-derived stem cells (hADSCs). (A) Morphological features of the fourth-generation hADSCs under light microscopy (scale bar $=75 \mathrm{~nm}$ ). (B) Representative images of Oil Red $O$ staining. (scale bar $=75 \mathrm{~nm}$ ). (C) the alkaline phosphatase calcium cobalt staining showed positive osteoblastic nodules (scale bar $=75 \mathrm{~nm}$ ). (D) Flow cytometry analysis of cell surface expression of CD29, CD90, CD31 and CD45 proteins in hADSCs. 
A

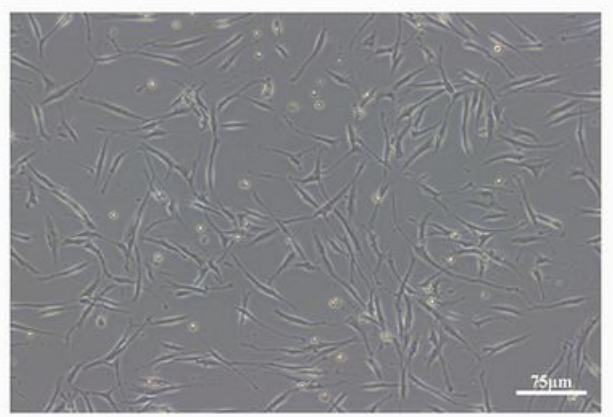

B

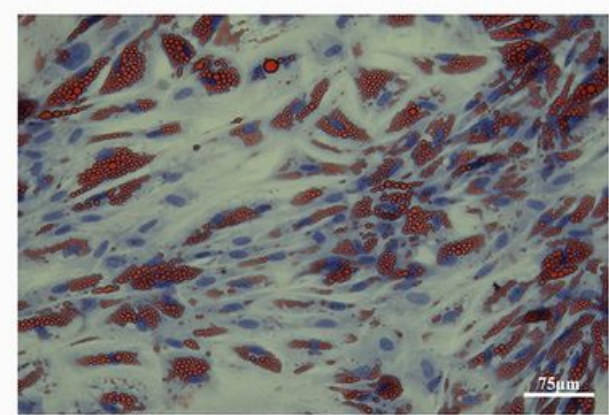

D
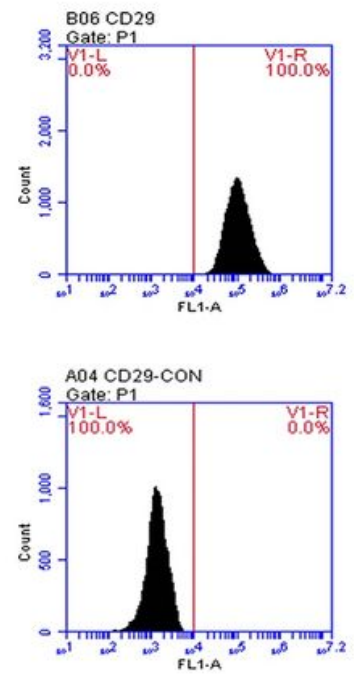
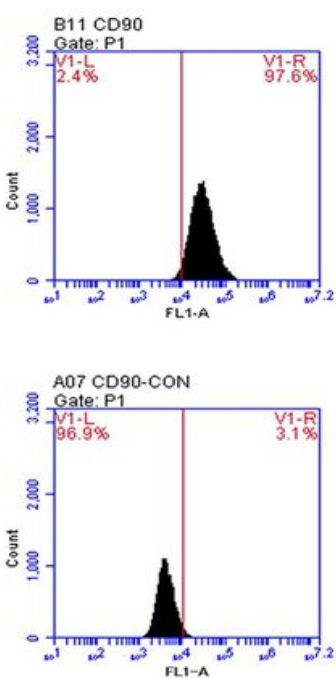
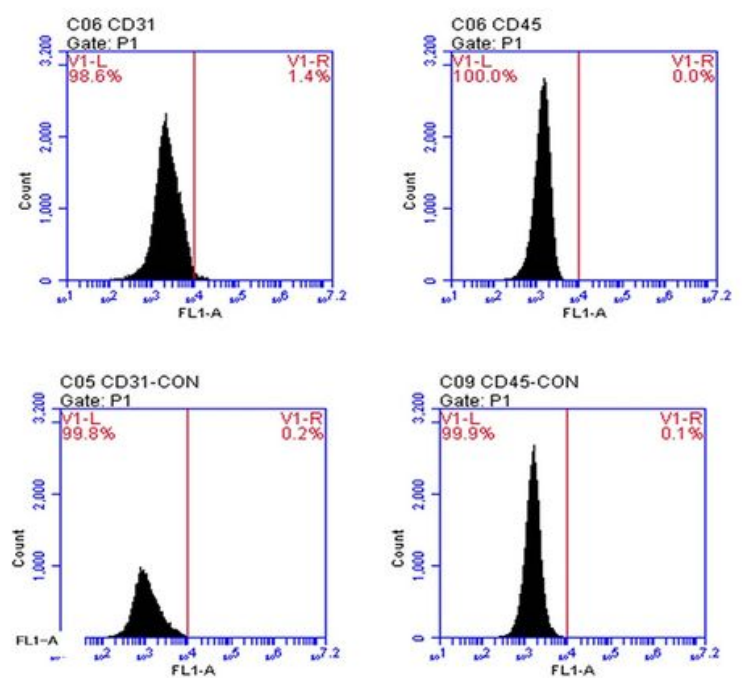

C

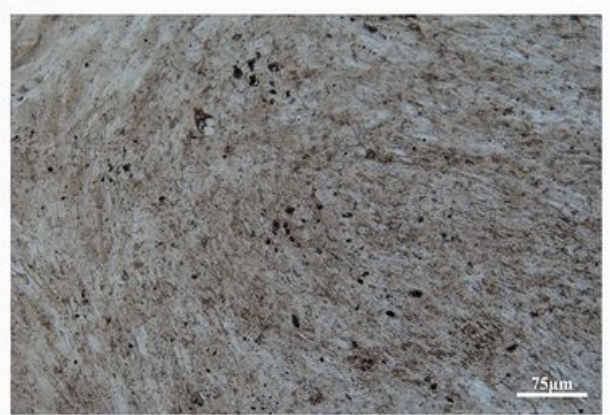

Figure 1

Characterizations of human adipose-derived stem cells (hADSCs). (A) Morphological features of the fourth-generation hADSCs under light microscopy (scale bar $=75 \mathrm{~nm}$ ). (B) Representative images of Oil Red $O$ staining. (scale bar $=75 \mathrm{~nm}$ ). (C) the alkaline phosphatase calcium cobalt staining showed positive osteoblastic nodules (scale bar $=75 \mathrm{~nm}$ ). (D) Flow cytometry analysis of cell surface expression of CD29, CD90, CD31 and CD45 proteins in hADSCs. 
A

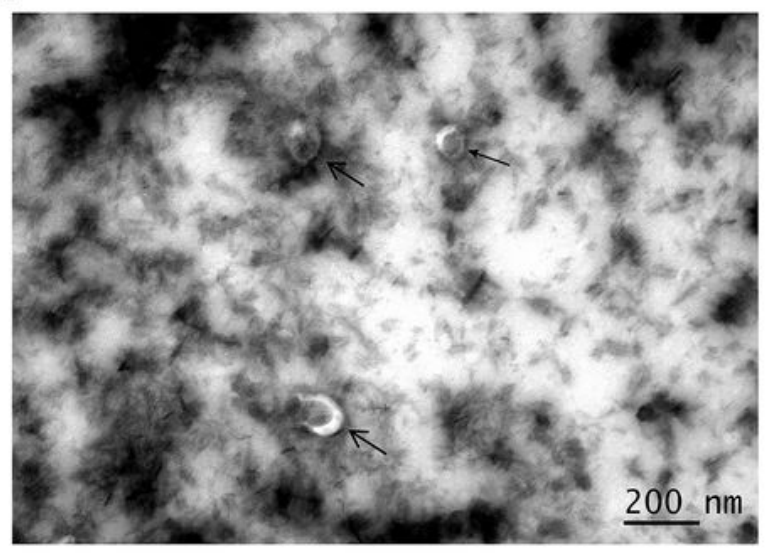

B

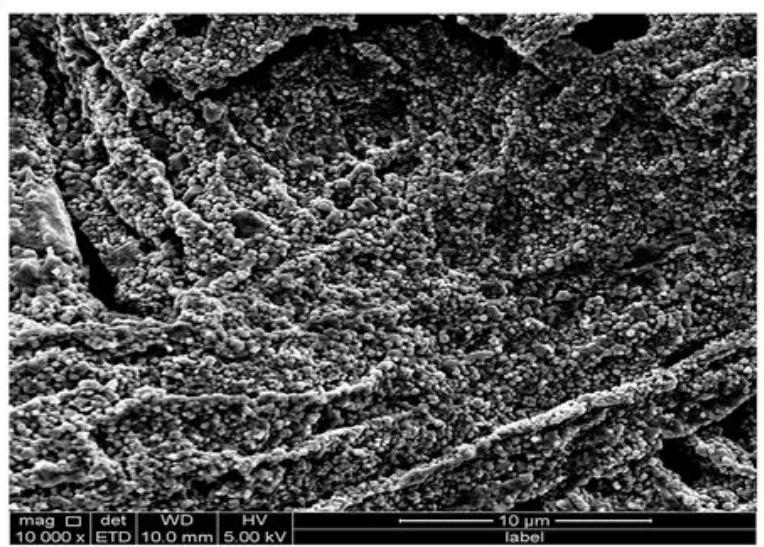

C
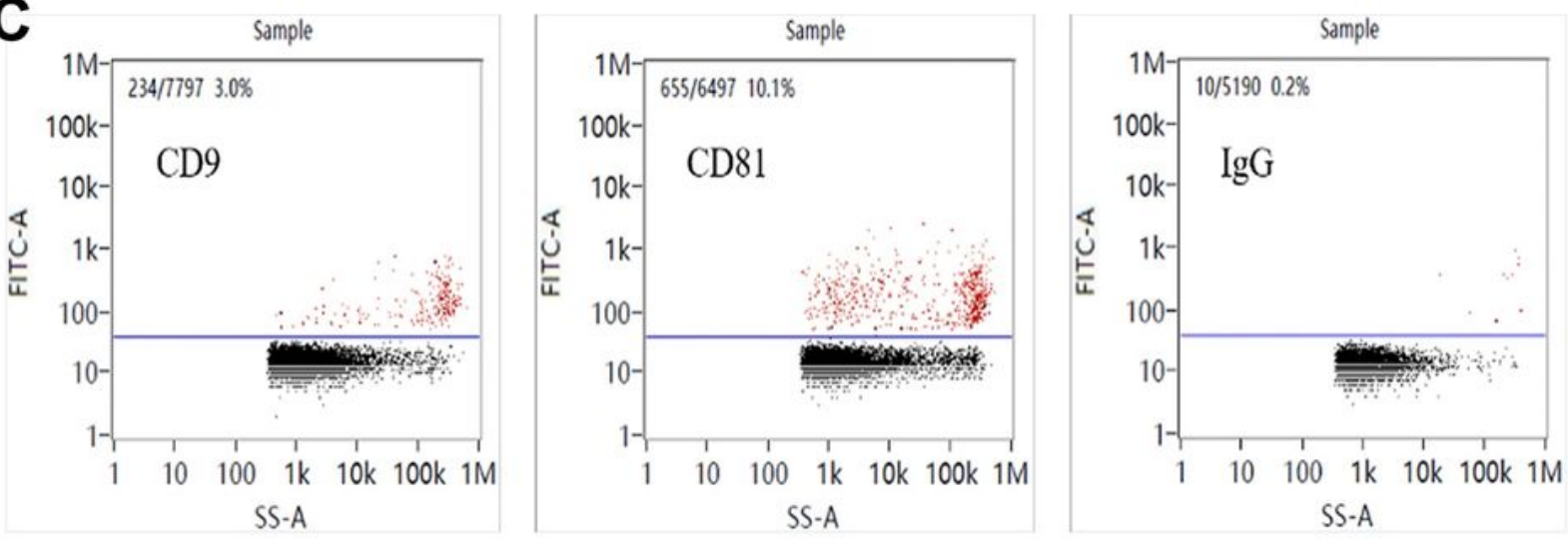

D

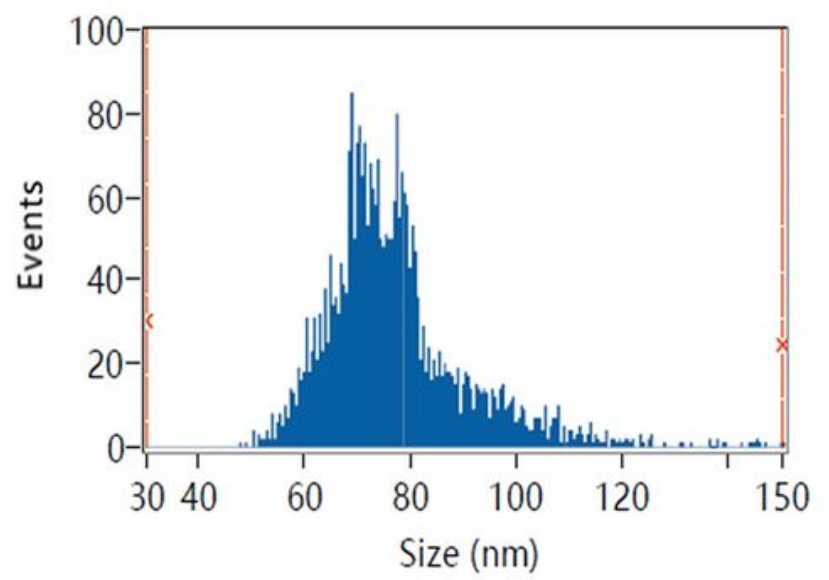

$\mathbf{E}$

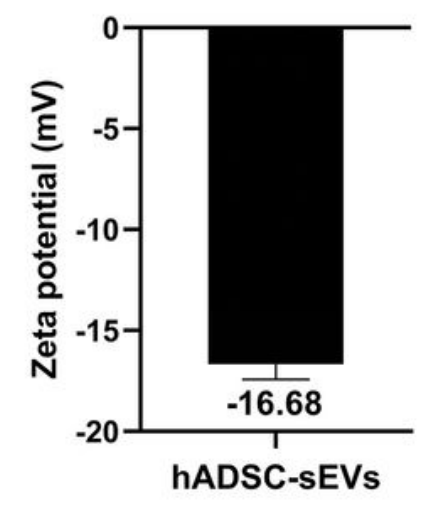

\section{Figure 2}

Characterizations of hADSC-sEVs. (A) Representative transmission electron microscopy image of ADSCsEVs with double circular vesicle structures was shown by directional arrow ( scale bar $=200 \mathrm{~nm}$ ). (B) Morphological characterization of sEVs with three-dimensional shapes under scanning electron microscopy (magnification 10,000x; scale bar $=10 \mathrm{um}$ ). (C) Nanoflow analysis of the hADSC-sEVs' 
surface protein marker (CD9, CD63 and CD81), and IgG as positive controls. (D) NTA measured the size distribution of sEVs. (E) The potential of hADSC-sEVs was measured by Zeta potential.

A

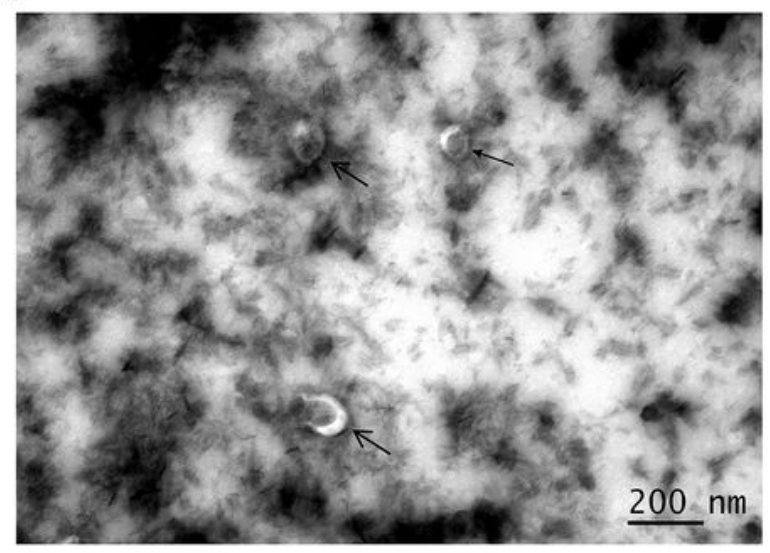

B

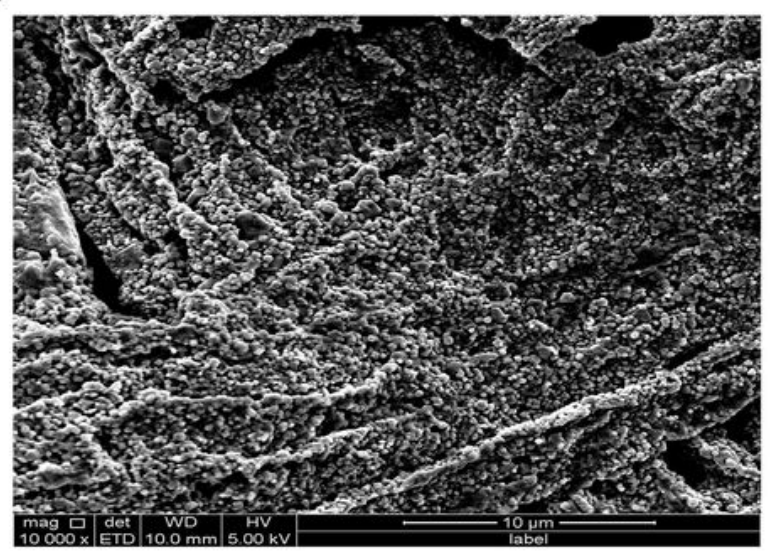

C

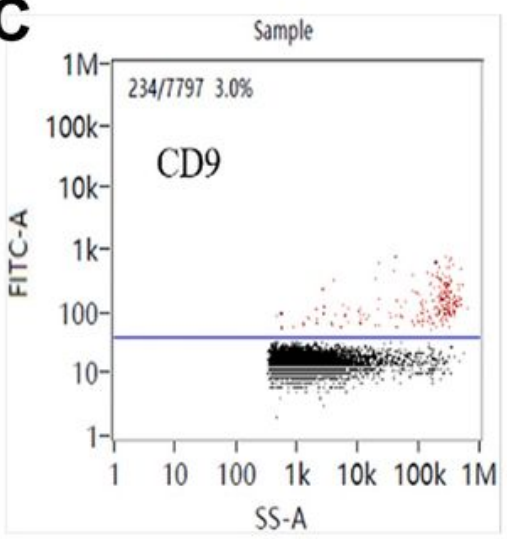

D

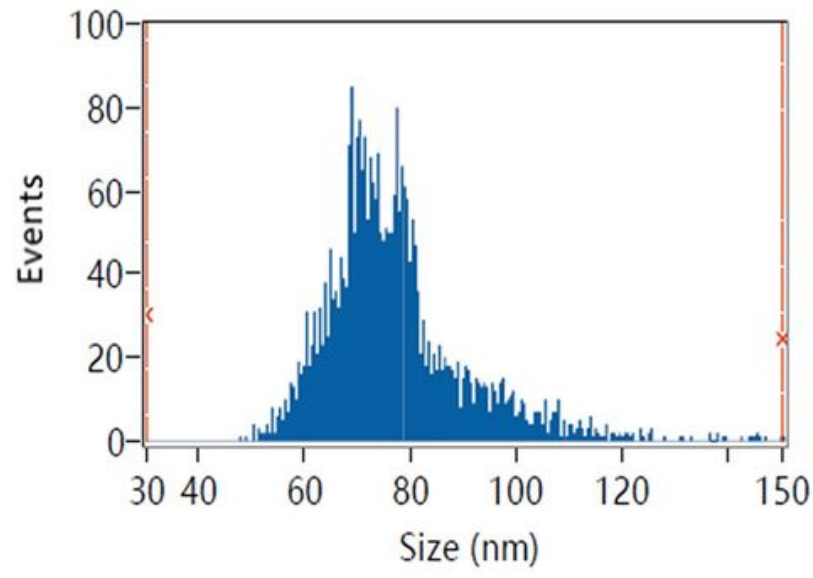

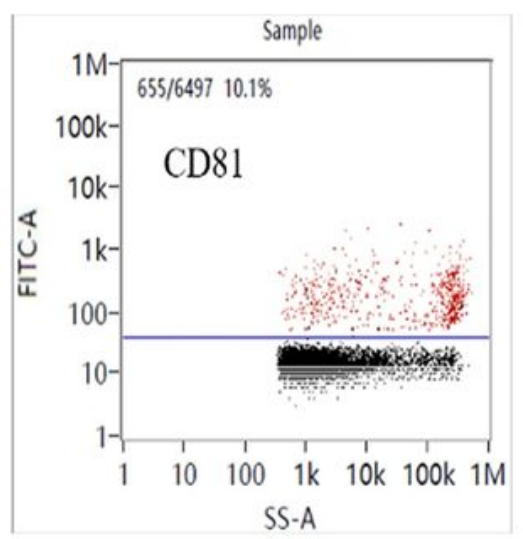

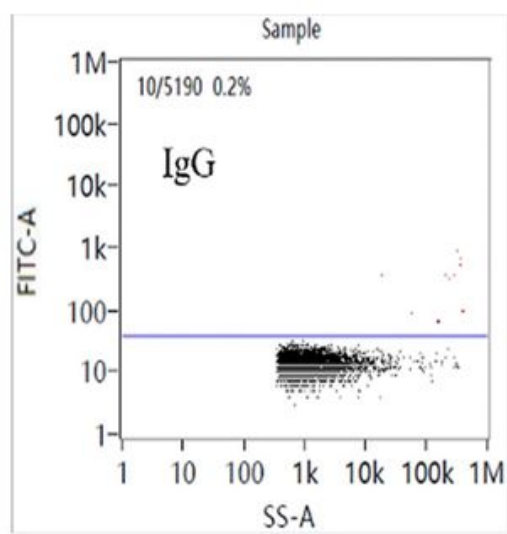

$\mathbf{E}$

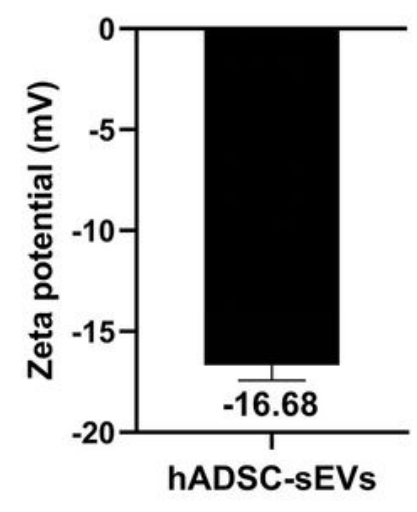

Figure 2

Characterizations of hADSC-sEVs. (A) Representative transmission electron microscopy image of ADSCsEVs with double circular vesicle structures was shown by directional arrow ( scale bar $=200 \mathrm{~nm}$ ). (B) Morphological characterization of sEVs with three-dimensional shapes under scanning electron 
microscopy (magnification 10,000x; scale bar $=10 \mathrm{um}$ ). (C) Nanoflow analysis of the hADSC-sEVs' surface protein marker (CD9, CD63 and CD81), and IgG as positive controls. (D) NTA measured the size distribution of sEVs. (E) The potential of hADSC-sEVs was measured by Zeta potential.
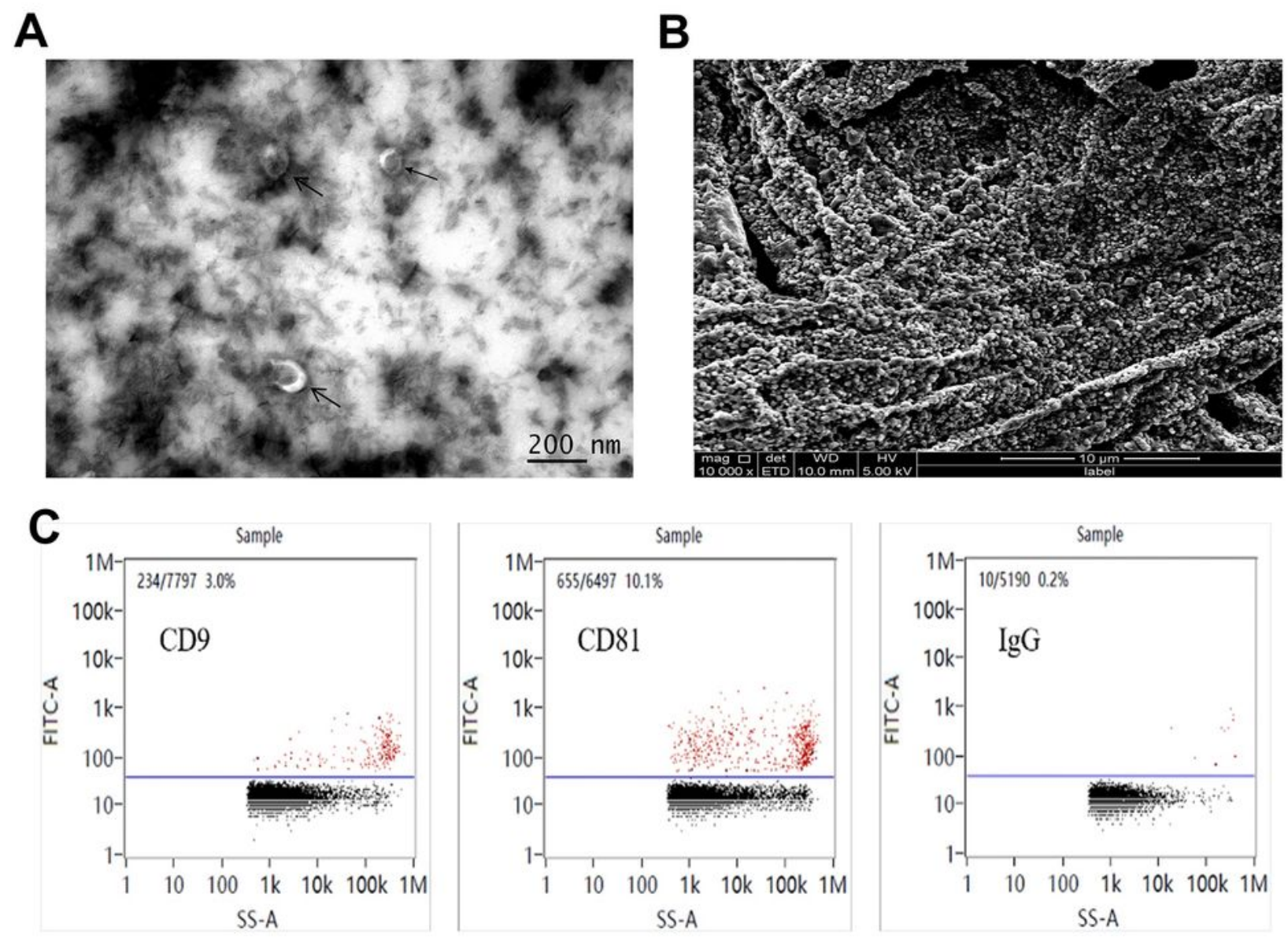

D

\section{$\mathbf{E}$}
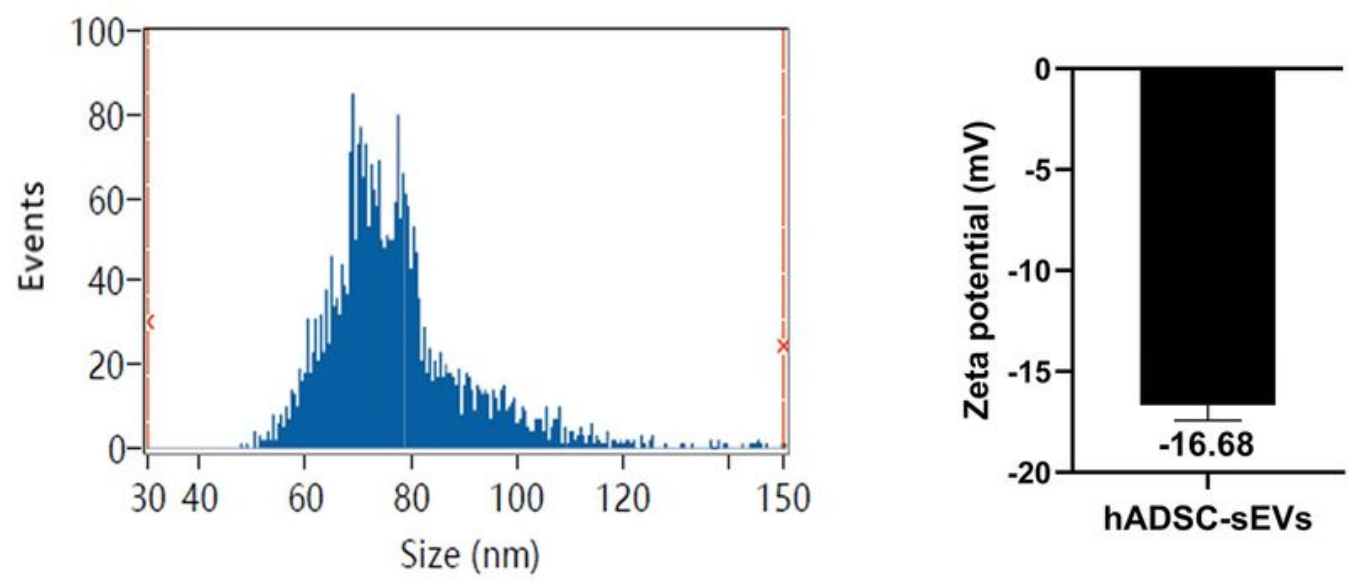

Figure 2

Characterizations of hADSC-sEVs. (A) Representative transmission electron microscopy image of ADSCsEVs with double circular vesicle structures was shown by directional arrow ( scale bar $=200 \mathrm{~nm}$ ). (B) 
Morphological characterization of sEVs with three-dimensional shapes under scanning electron microscopy (magnification 10,000x; scale bar $=10 \mathrm{um}$ ). (C) Nanoflow analysis of the hADSC-sEVs' surface protein marker (CD9, CD63 and CD81), and IgG as positive controls. (D) NTA measured the size distribution of sEVs. (E) The potential of hADSC-sEVs was measured by Zeta potential.

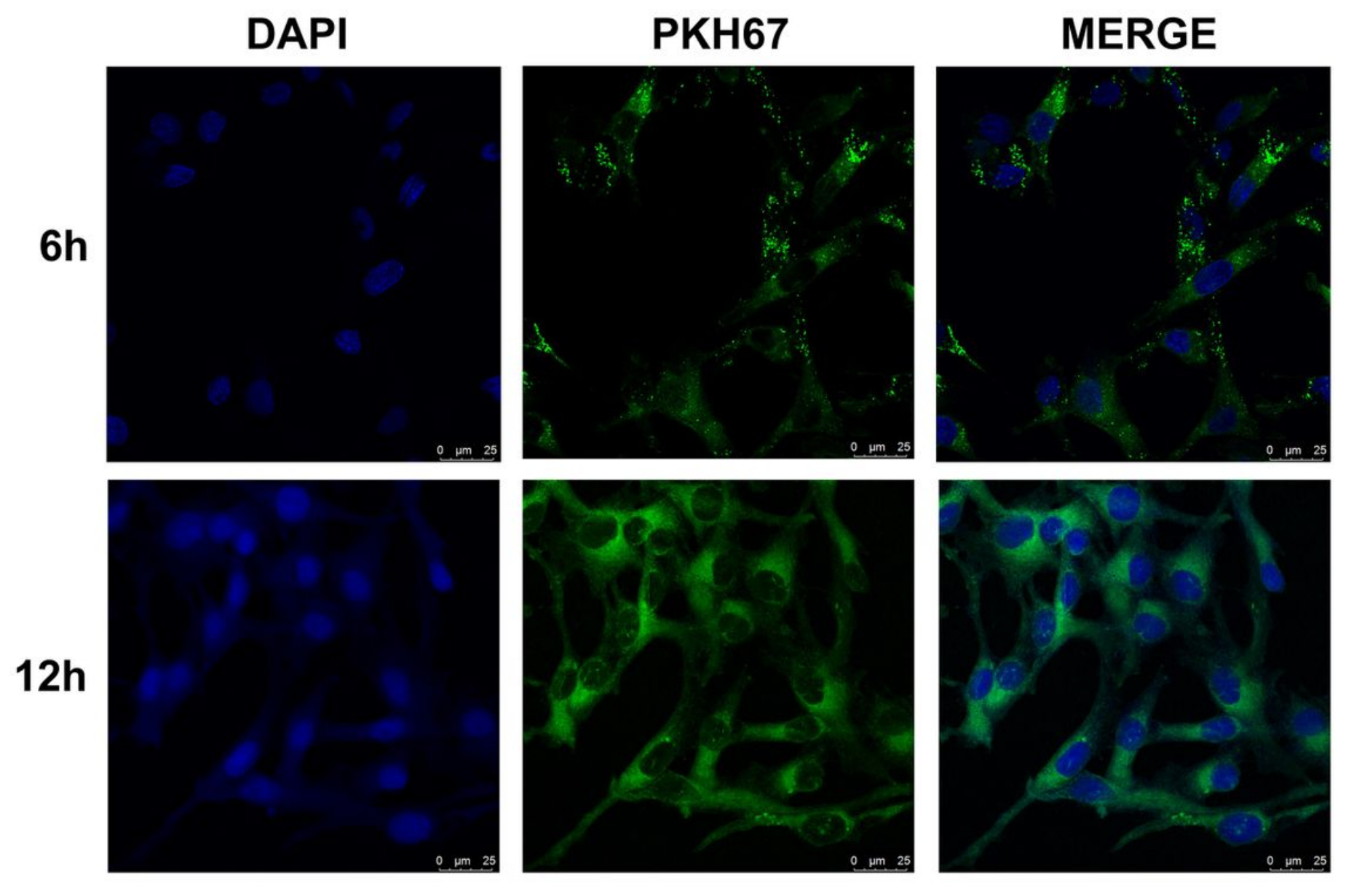

Figure 3

hADSC-sEVs internalization to HUVECs. hADSC-sEVs (labelled with PKH67 dye, green) and HUVECs (nuclei stained with DAPI) were co-cultured for $6 \mathrm{~h}$ and $12 \mathrm{~h}$, respectively. Representative fluorescence images were shown above. 


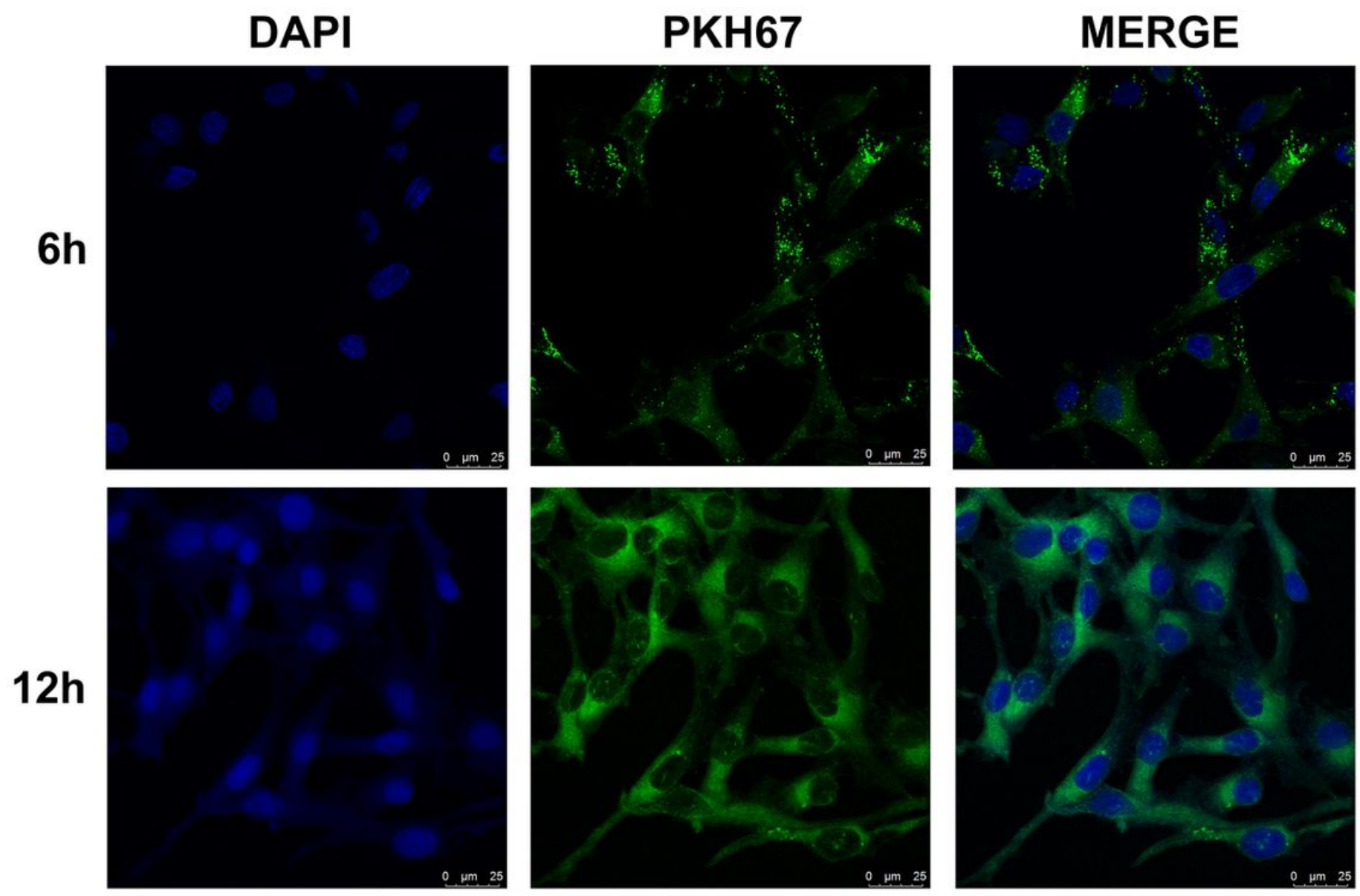

Figure 3

hADSC-sEVs internalization to HUVECs. hADSC-sEVs (labelled with PKH67 dye, green) and HUVECs (nuclei stained with DAPI) were co-cultured for $6 \mathrm{~h}$ and $12 \mathrm{~h}$, respectively. Representative fluorescence images were shown above. 


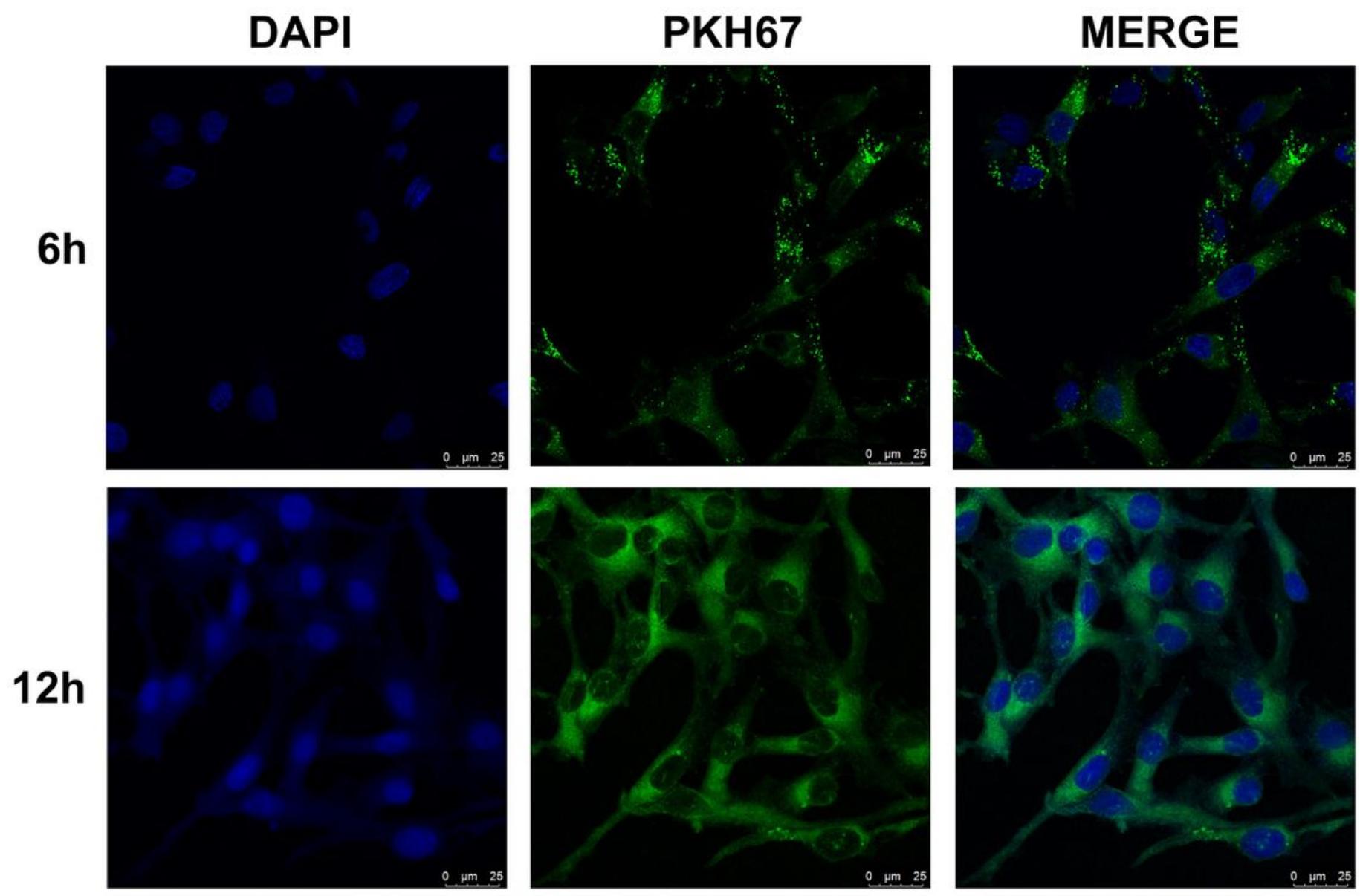

Figure 3

hADSC-sEVs internalization to HUVECs. hADSC-sEVs (labelled with PKH67 dye, green) and HUVECs (nuclei stained with DAPI) were co-cultured for $6 \mathrm{~h}$ and $12 \mathrm{~h}$, respectively. Representative fluorescence images were shown above. 

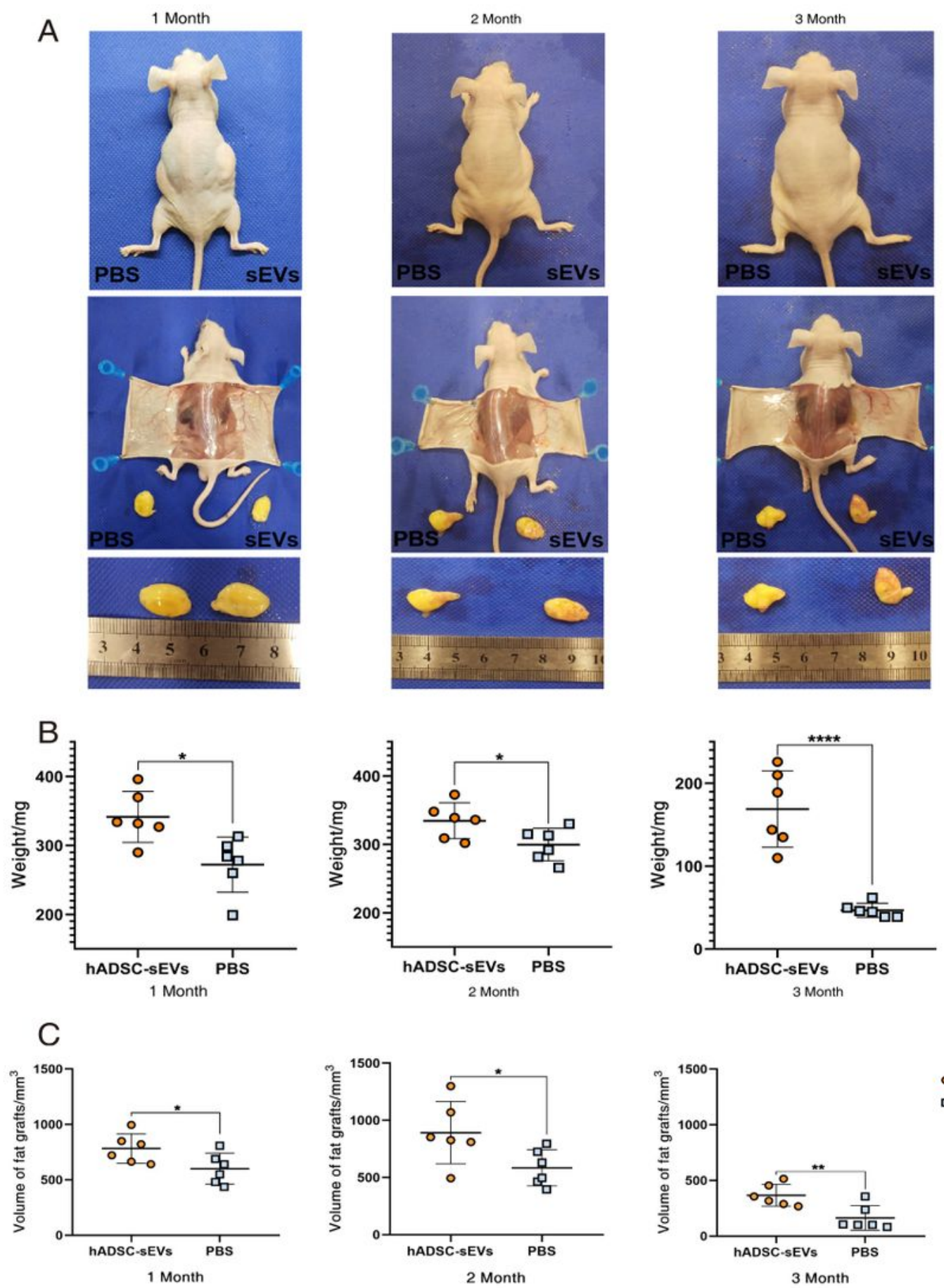

- hADSC-sEVs

口 PBS

\section{Figure 4}

hADSC-sEVs improved the fat graft survival rate in the nude mice model. (A) Gross observation of the grafted fat demonstrated that the hADSC-sEVs group had larger graft sizes compared to that in the control group. (B, C) 1, 2 and 3 months after fat grafting, the weight and volume of grafted fat were significantly greater in the hADSC-sEVs group compared to the control group $(*, p<0.05, * \star, p<0.01$, $\star \star \star *$, $p<0.001, * \star \star \star, ~ p<0.0001)$. 

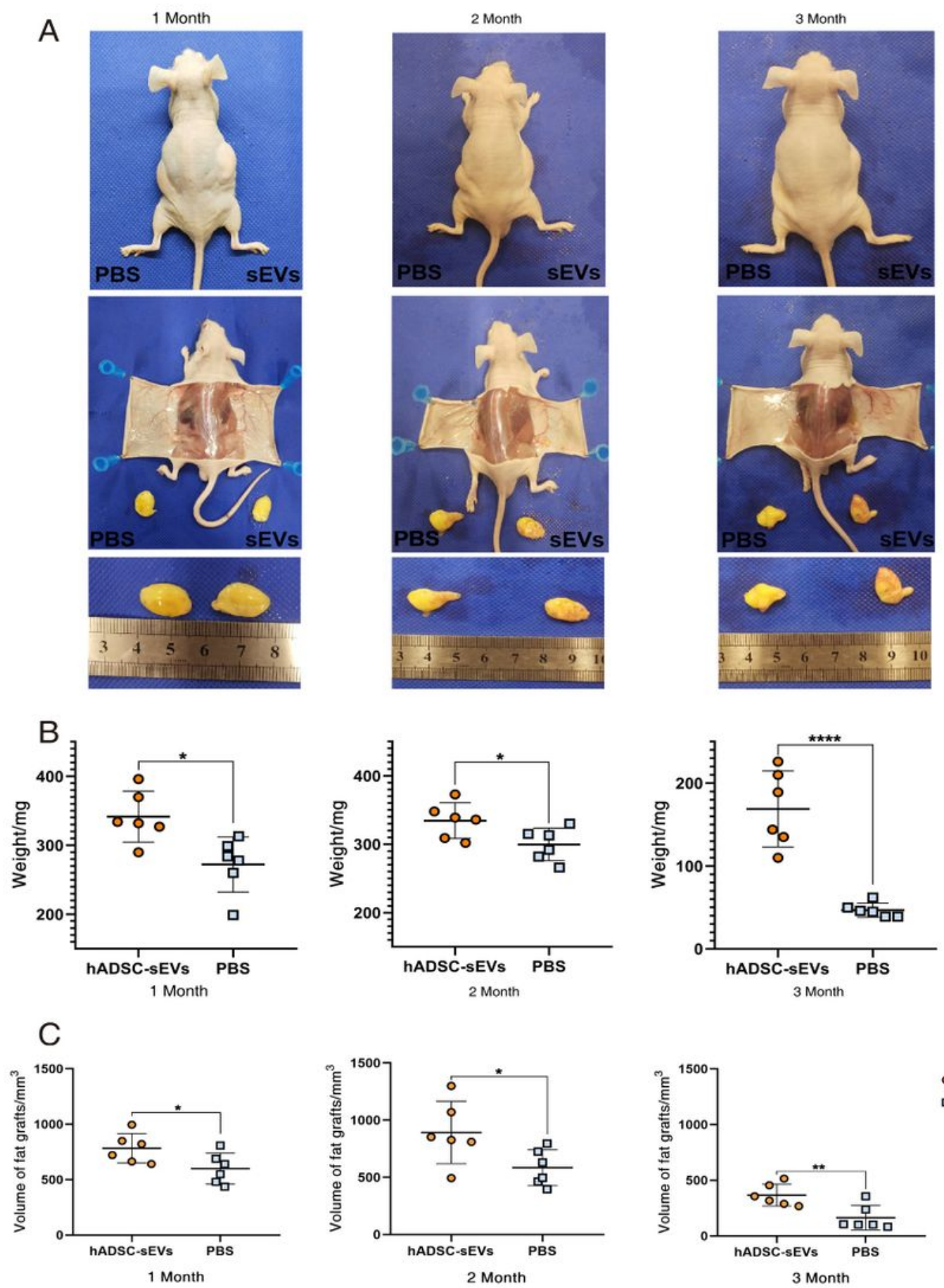

- hADSC-sEVs

- PBS

\section{Figure 4}

hADSC-sEVs improved the fat graft survival rate in the nude mice model. (A) Gross observation of the grafted fat demonstrated that the hADSC-sEVs group had larger graft sizes compared to that in the control group. (B, C) 1, 2 and 3 months after fat grafting, the weight and volume of grafted fat were significantly greater in the hADSC-sEVs group compared to the control group $(*, p<0.05, * \star, p<0.01$, $\star \star \star *$, $p<0.001, * \star \star \star, p<0.0001)$. 

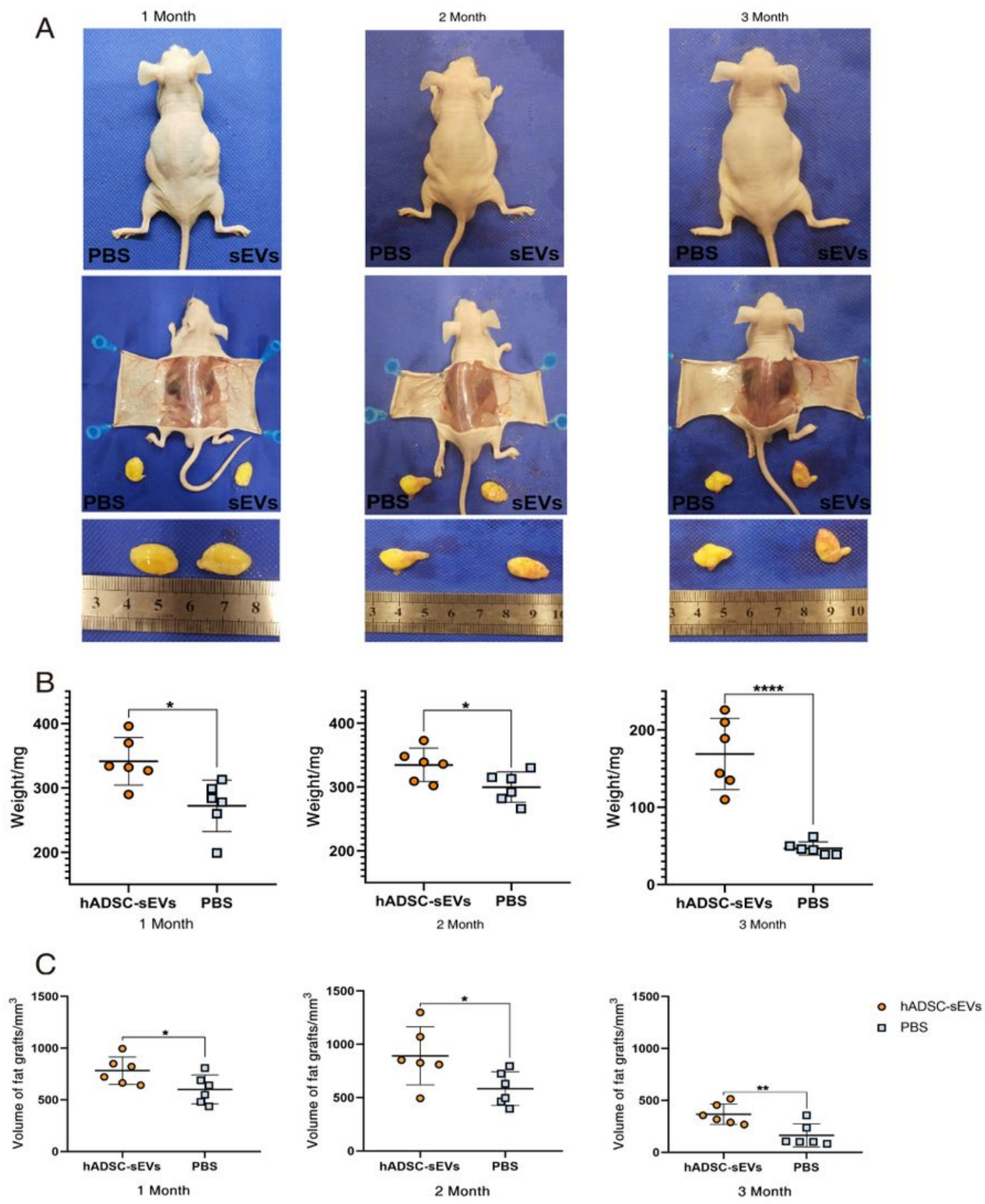

\section{Figure 4}

hADSC-sEVs improved the fat graft survival rate in the nude mice model. (A) Gross observation of the grafted fat demonstrated that the hADSC-sEVs group had larger graft sizes compared to that in the control group. (B, C) 1, 2 and 3 months after fat grafting, the weight and volume of grafted fat were significantly greater in the hADSC-sEVs group compared to the control group $(*, p<0.05, * \star, p<0.01$, $\star \star \star *$, $p<0.001, * \star \star \star, ~ p<0.0001)$. 
A

1 month

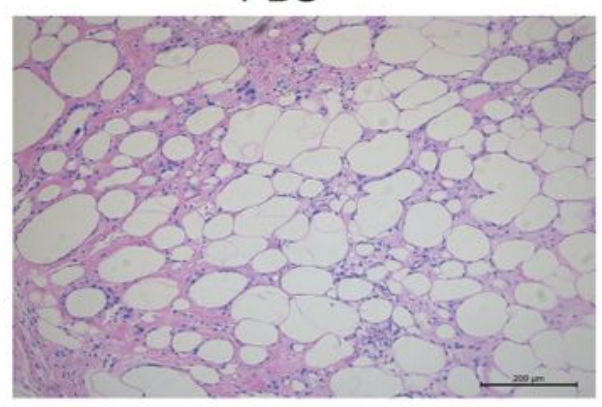

PBS

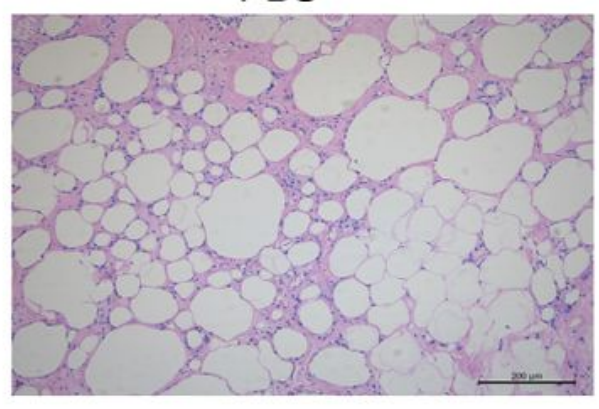

2 month
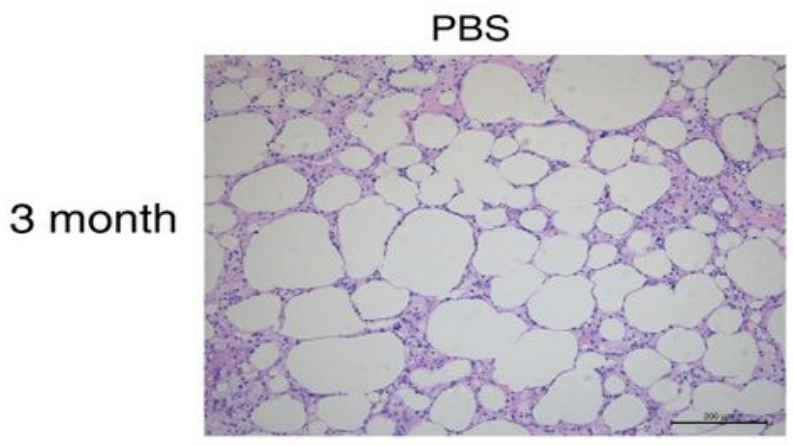

hADSC-sEVS

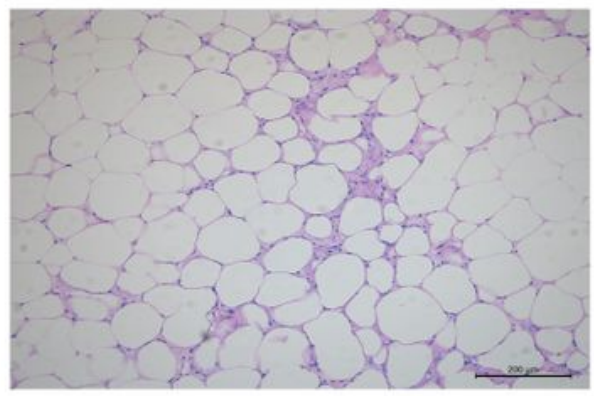

hADSC-sEVs

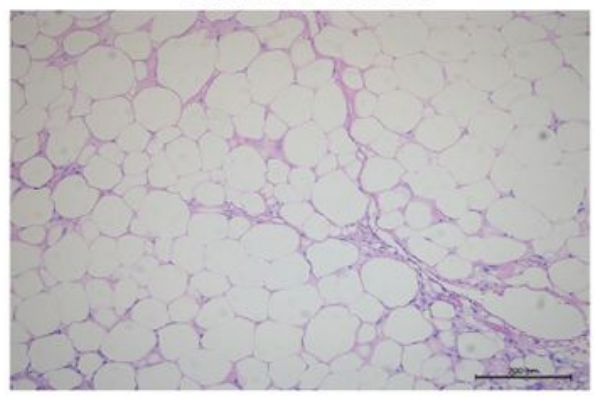

hADSC-sEVs

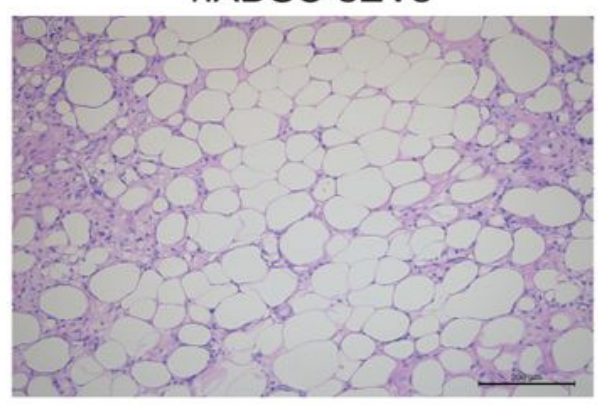

B

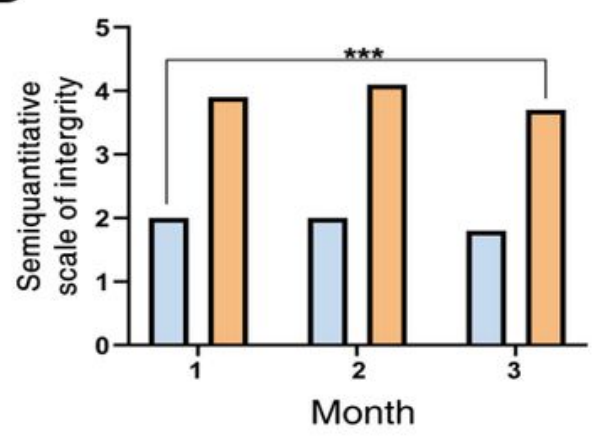

$\mathrm{C}$

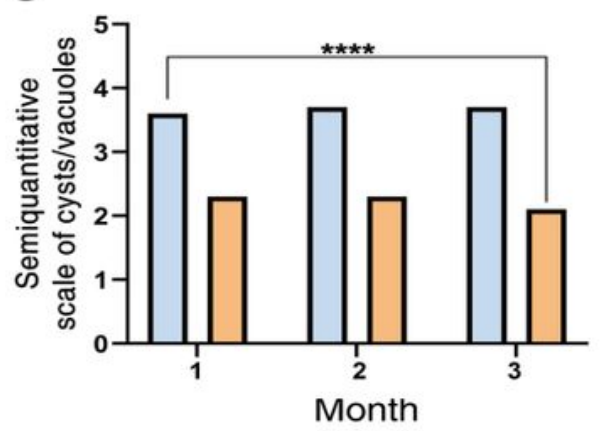

$\square$ PBS
$\square$ hADSC-sEVs

\section{Figure 5}

Histological evaluation of HE staining of the fat graft. (A) HE staining revealed that the grafted fat in the hADSC-sEVs groups exhibited better survival and morphologic integrity compared to the control group. (B, C) Semiquantitative scale of integrity and cysts/vacuoles of HE staining( $\left(\star \star \star, p<0.001^{\star \star \star \star}, p<0.0001\right)$. 
A

1 month
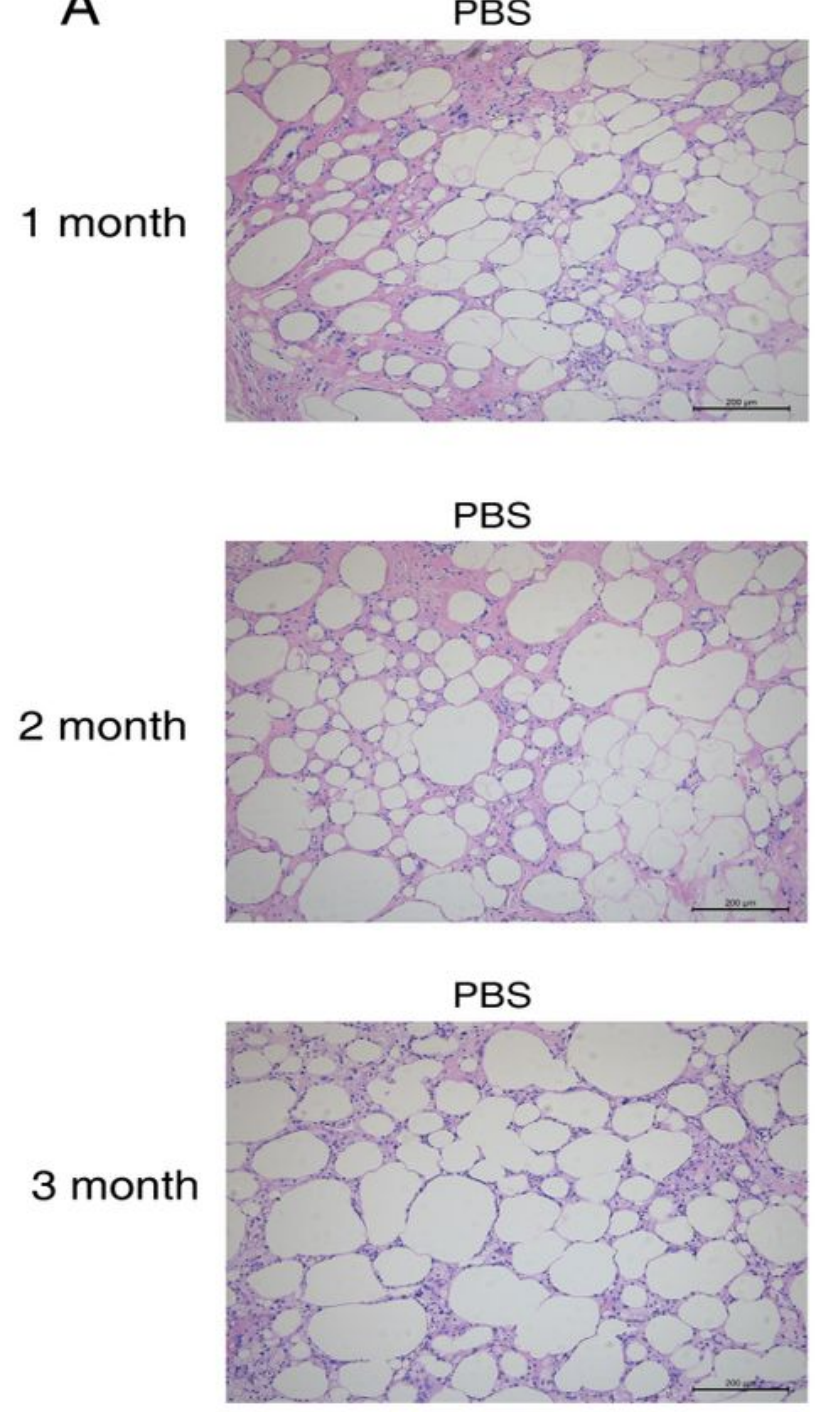

hADSC-sEVs

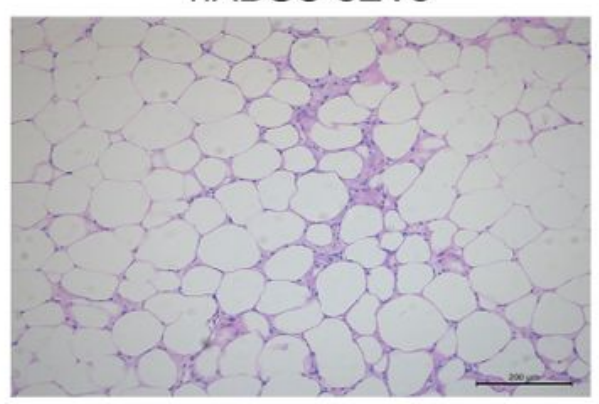

hADSC-sEVs

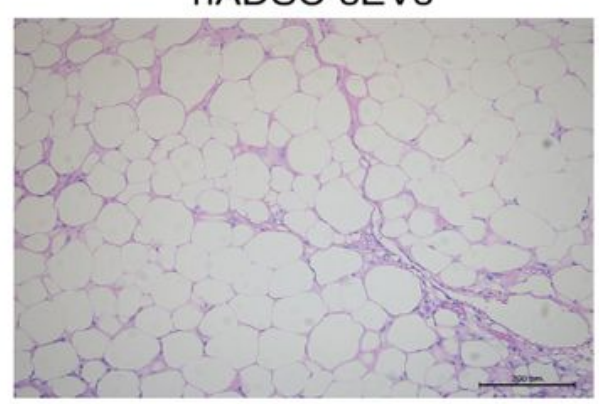

hADSC-sEVs

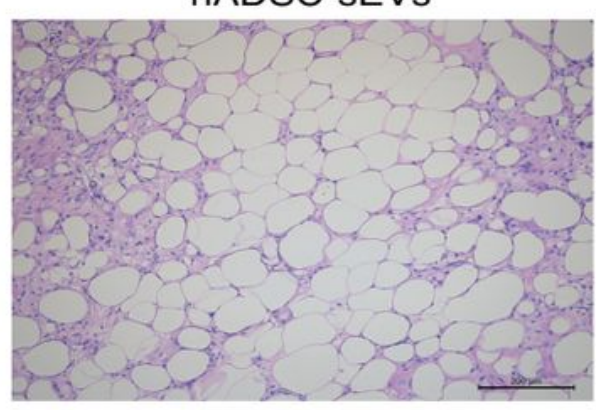

B

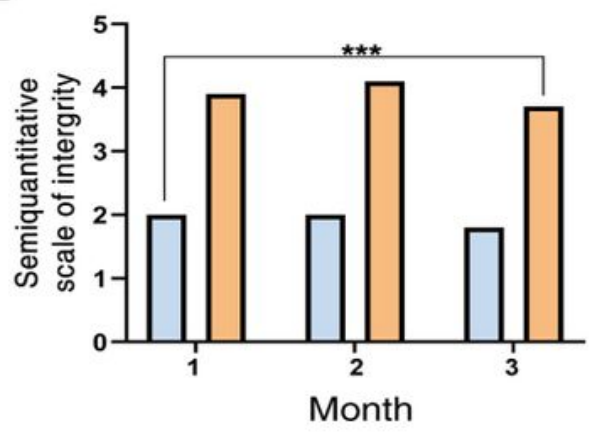

C

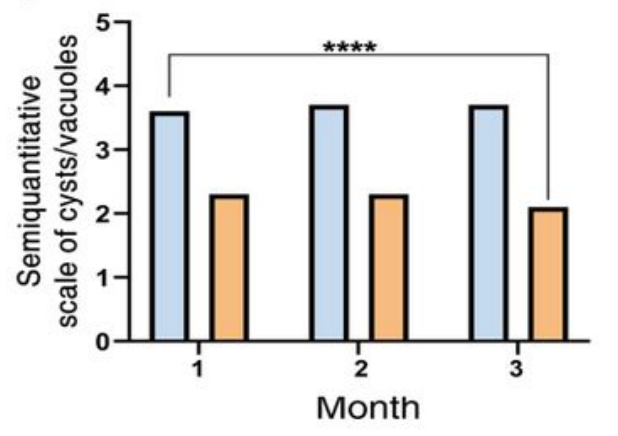

$\square$ PBS
$\square$ hADSC-sEVS

\section{Figure 5}

Histological evaluation of HE staining of the fat graft. (A) HE staining revealed that the grafted fat in the hADSC-sEVs groups exhibited better survival and morphologic integrity compared to the control group. (B, C) Semiquantitative scale of integrity and cysts/vacuoles of HE staining( $\left(\star \star \star, p<0.001^{\star \star \star \star}, p<0.0001\right)$. 


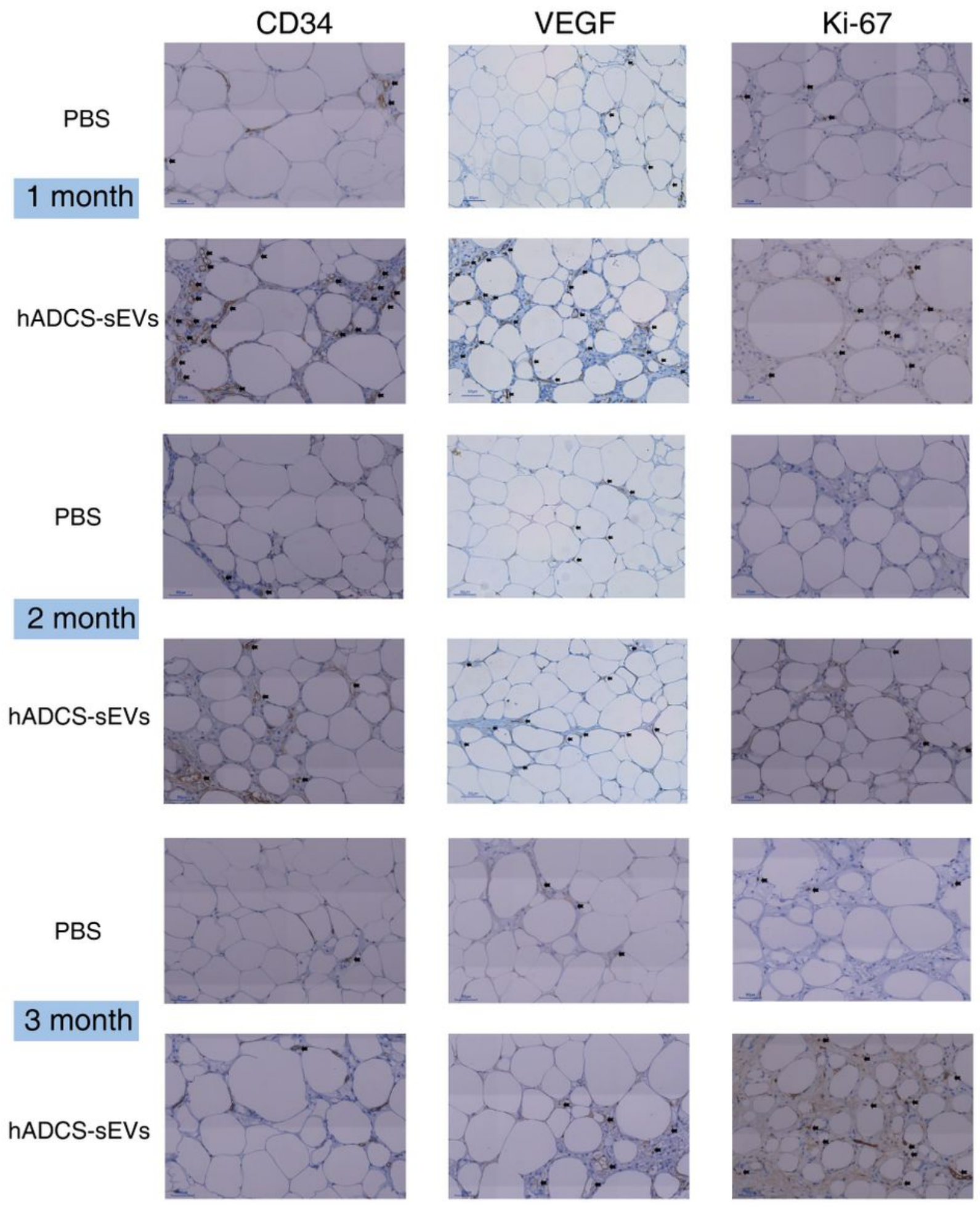

\section{Figure 6}

hADSC-sEVs promoted neovascularization in the nude mice fat grafting model. Immunohistochemical staining to CD34『VEGF and Ki-67 in grafted fat from different weeks. CD34 was selectively expressed in the small vessel endothelium. VEGF was mainly localized in endothelial cells and stromal cells. Signs of proliferation (nuclei stained by Ki-67) in the vessel endothelium ( scale bar $=50 \mu \mathrm{m}$ ) 


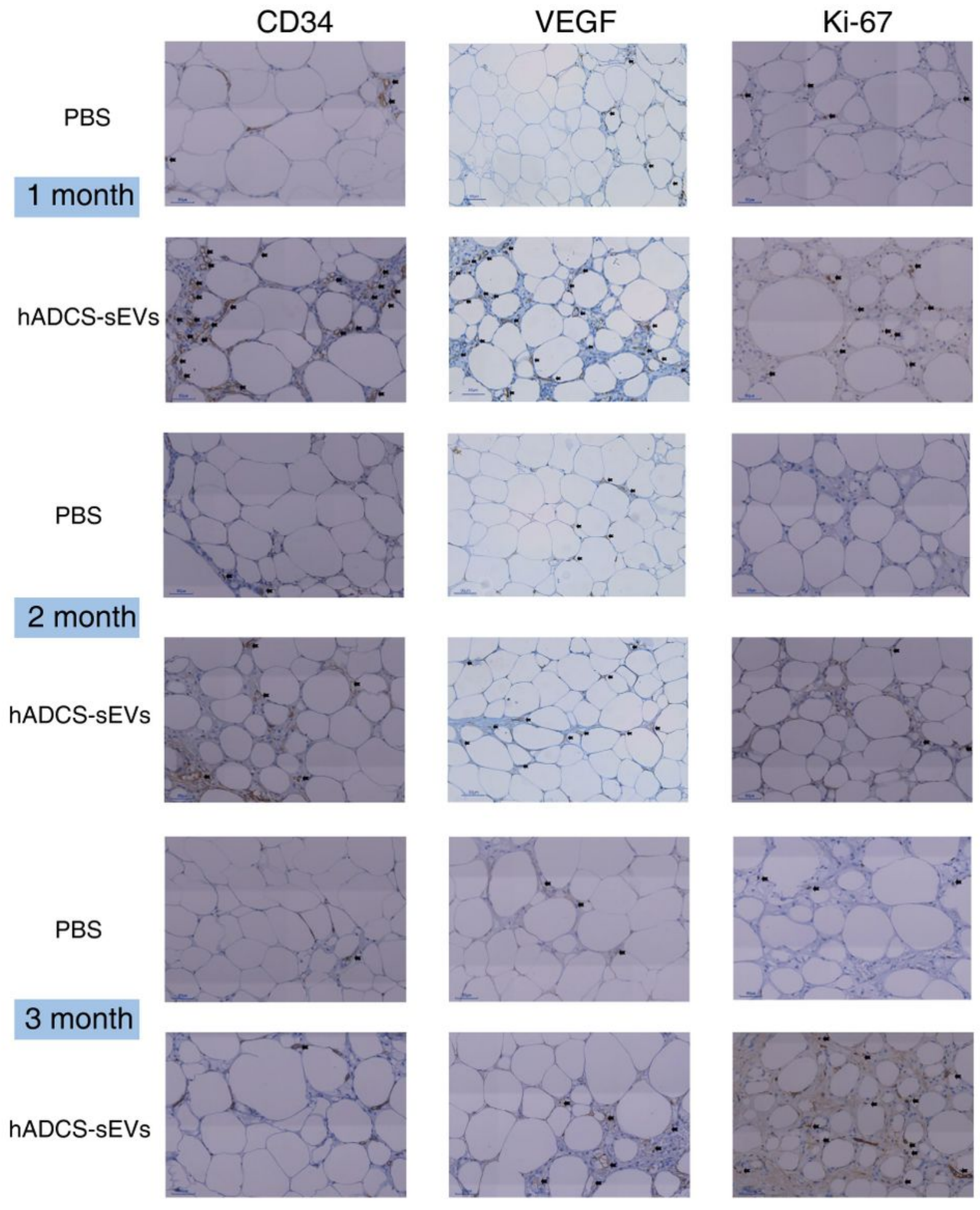

\section{Figure 6}

hADSC-sEVs promoted neovascularization in the nude mice fat grafting model. Immunohistochemical staining to CD34『VEGF and Ki-67 in grafted fat from different weeks. CD34 was selectively expressed in the small vessel endothelium. VEGF was mainly localized in endothelial cells and stromal cells. Signs of proliferation (nuclei stained by Ki-67) in the vessel endothelium ( scale bar $=50 \mu \mathrm{m}$ ) 


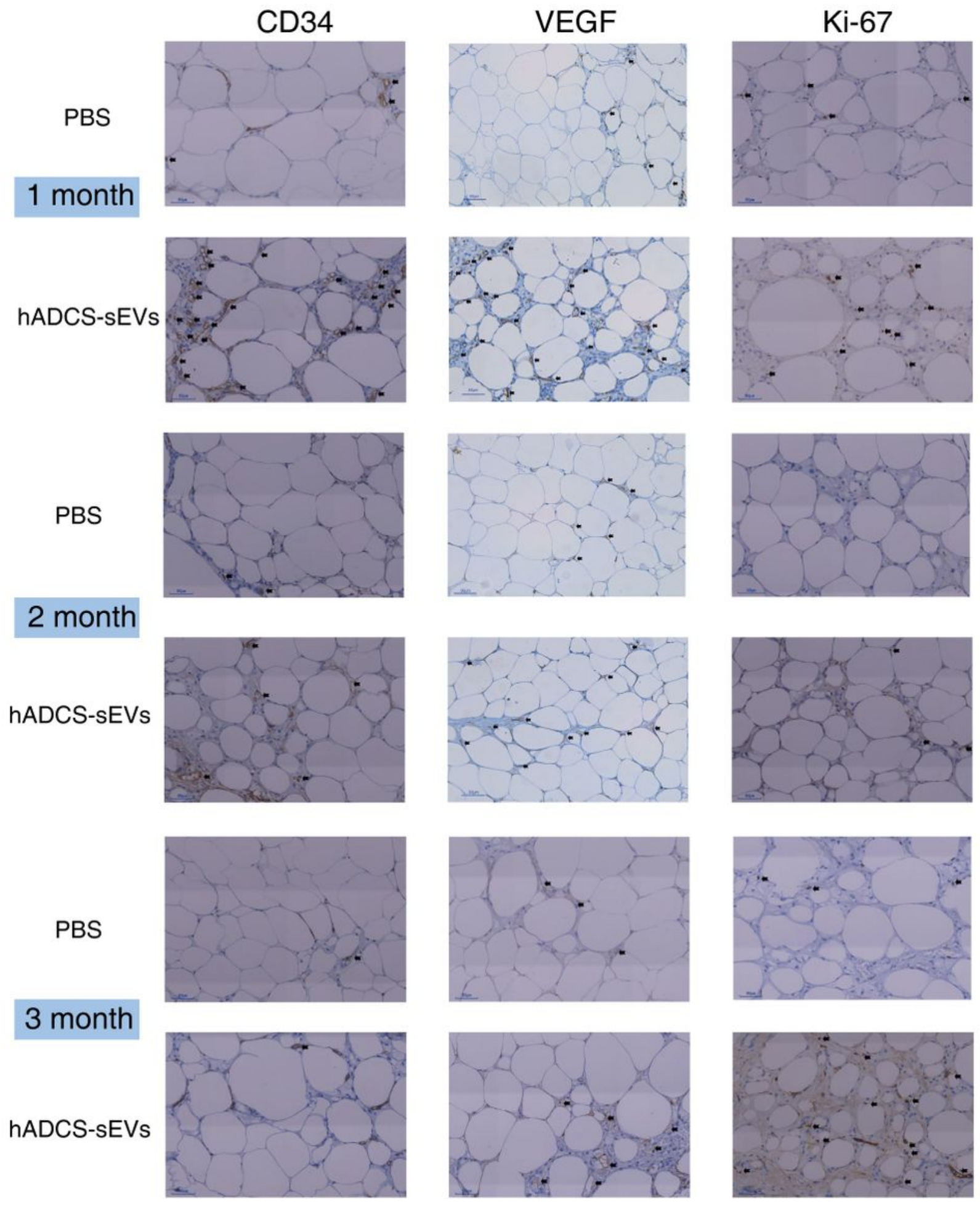

\section{Figure 6}

hADSC-sEVs promoted neovascularization in the nude mice fat grafting model. Immunohistochemical staining to CD34『VEGF and Ki-67 in grafted fat from different weeks. CD34 was selectively expressed in the small vessel endothelium. VEGF was mainly localized in endothelial cells and stromal cells. Signs of proliferation (nuclei stained by Ki-67) in the vessel endothelium ( scale bar $=50 \mu \mathrm{m}$ ) 
A

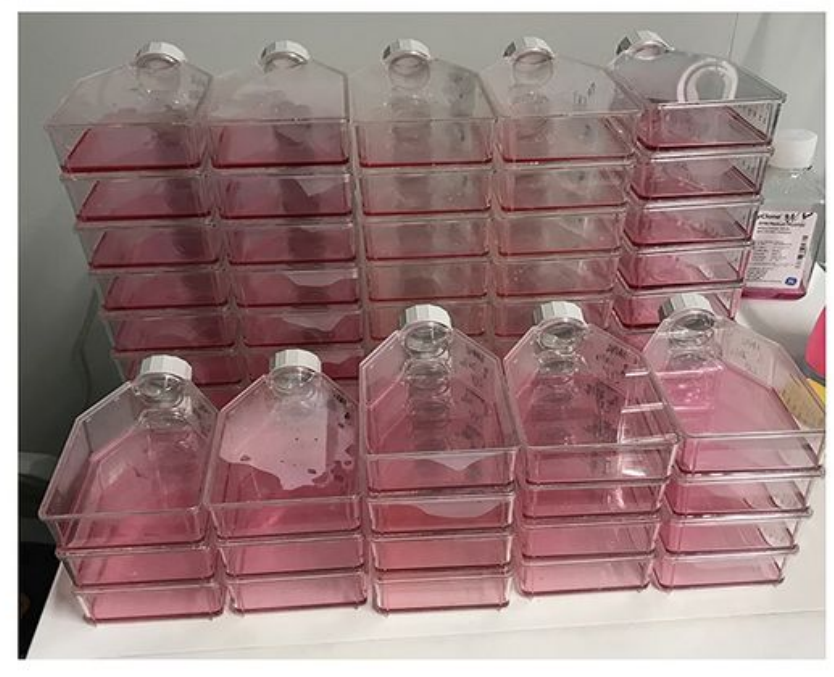

C

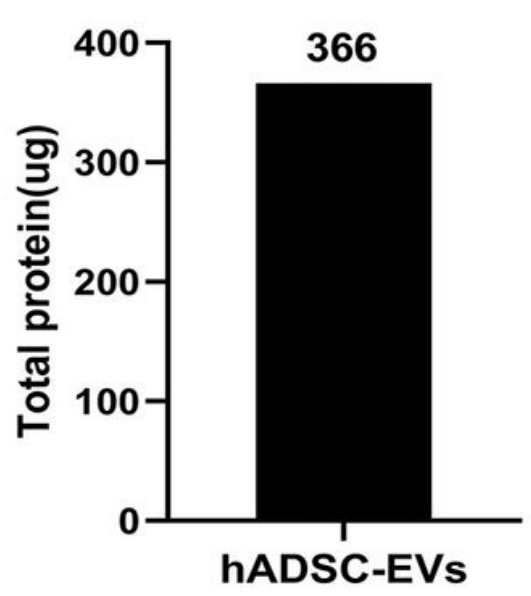

B

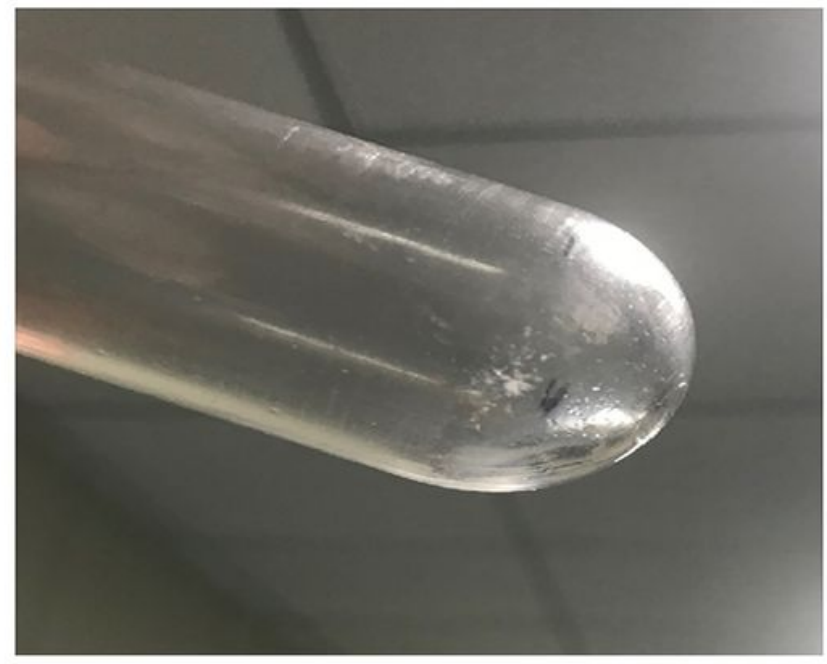

D

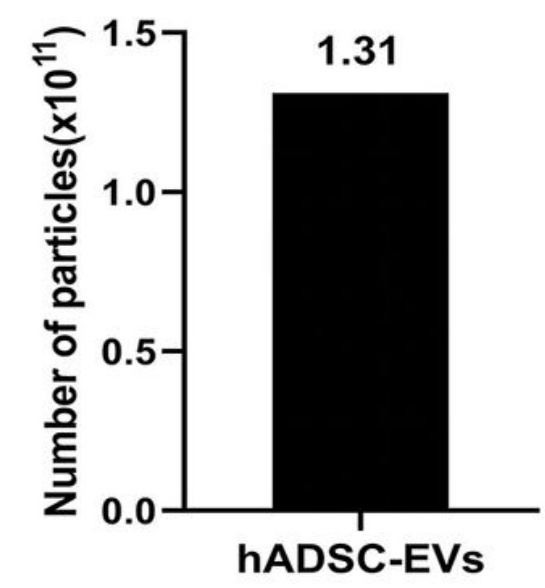

Figure 7

The small extracellular vesicles yield of supernatant from hADSCs. (A) General view: the number of bottles for culturing cells at one time. (B) Macroscopic view of sEVs obtained from figure 6A. $(C, D)$ The total protein $(\mathrm{ug})$ and the number of particles $(\times 1011)$ of sEVs from $3 \times 108 \mathrm{hADSC}$. 
A

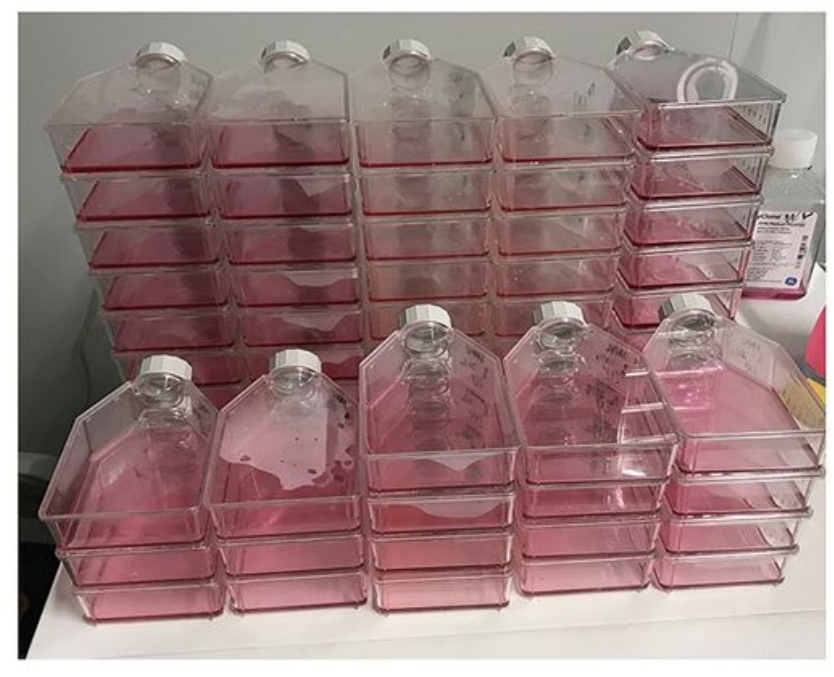

C

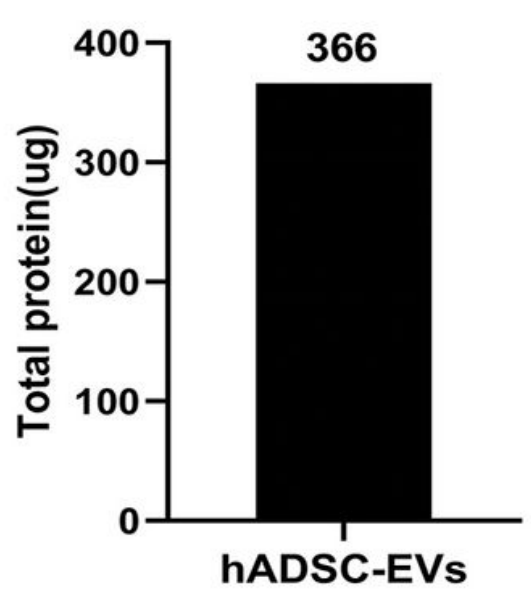

B

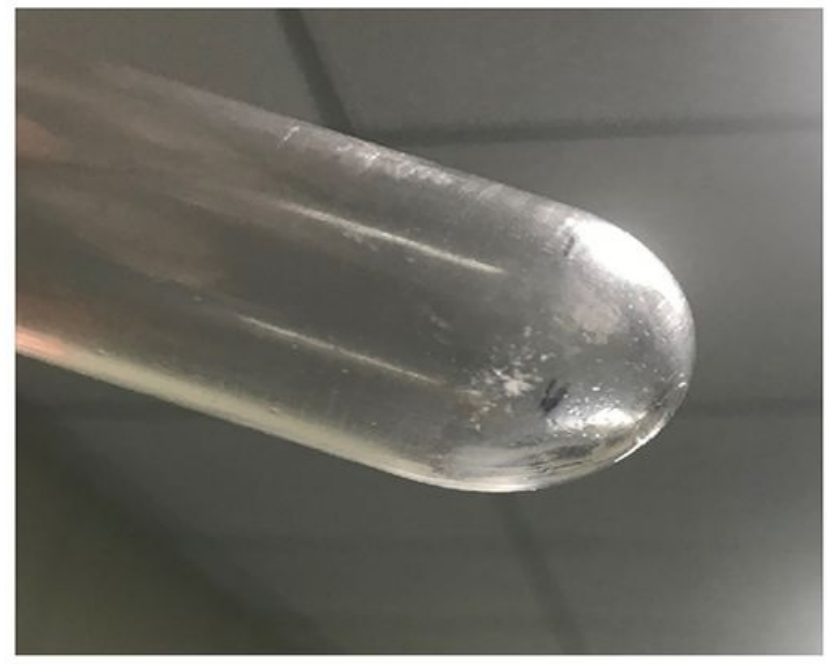

D

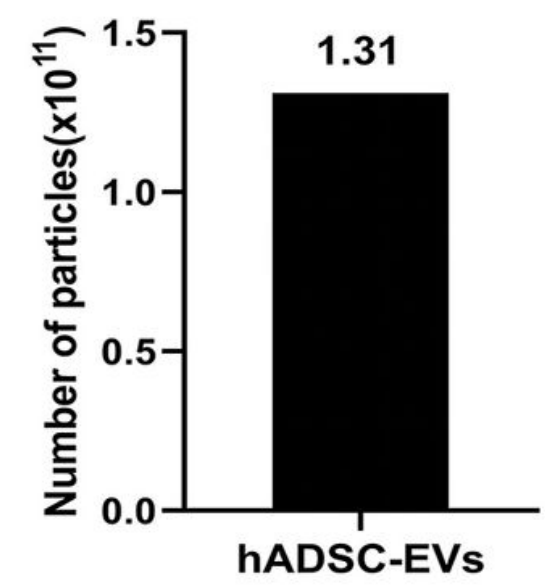

Figure 7

The small extracellular vesicles yield of supernatant from hADSCs. (A) General view: the number of bottles for culturing cells at one time. (B) Macroscopic view of sEVs obtained from figure 6A. $(C, D)$ The total protein $(\mathrm{ug})$ and the number of particles $(\times 1011)$ of sEVs from $3 \times 108 \mathrm{hADSC}$. 
A

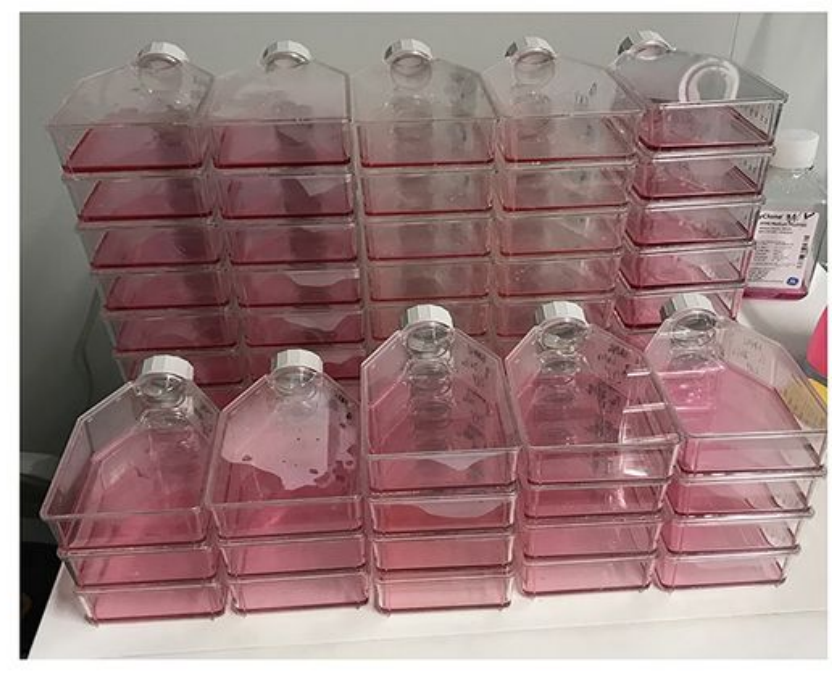

C

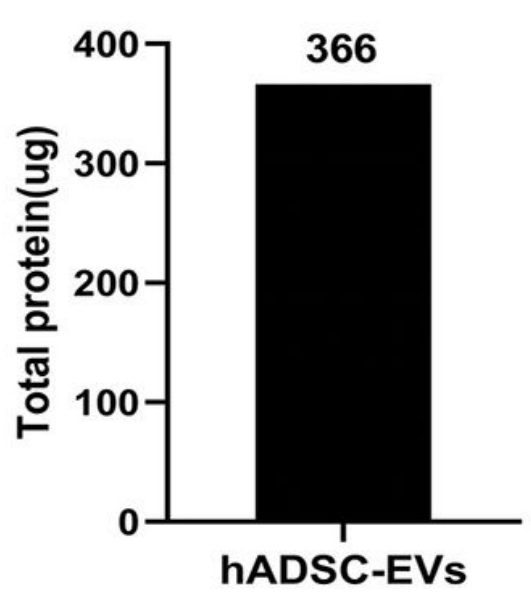

B

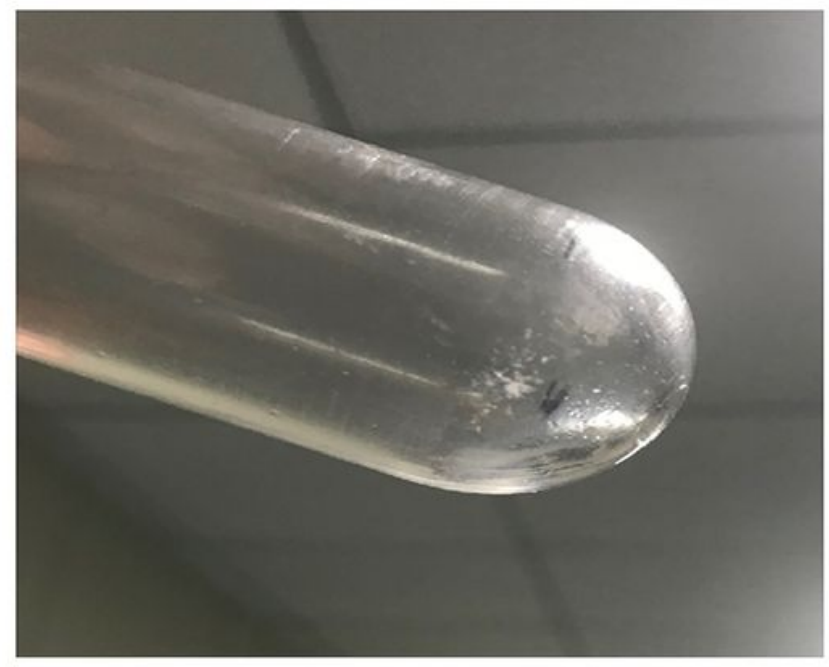

D

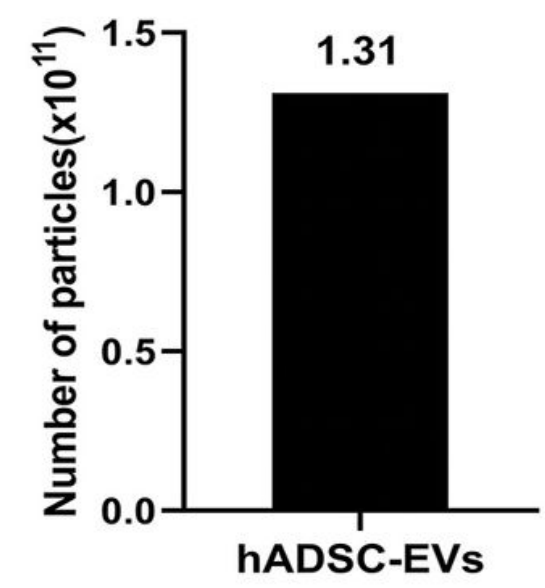

Figure 7

The small extracellular vesicles yield of supernatant from hADSCs. (A) General view: the number of bottles for culturing cells at one time. (B) Macroscopic view of sEVs obtained from figure 6A. $(C, D)$ The total protein $(\mathrm{ug})$ and the number of particles $(\times 1011)$ of sEVs from $3 \times 108 \mathrm{hADSC}$. 